\title{
Understanding the Scatter in the Spatially-resolved Star Formation Main Sequence of Local Massive Spiral Galaxies
}

\author{
Abdurro'uf, ${ }^{1 \star}$ Masayuki Akiyama, ${ }^{1}$ \\ ${ }^{1}$ Astronomical Institute, Tohoku University, Aramaki, Aoba, Sendai 980-8578, Japan
}

Accepted XXX. Received YYY; in original form ZZZ

\begin{abstract}
We investigate the relation between star formation rate (SFR) and stellar mass $\left(M_{*}\right)$ in sub-galactic $(\sim 1 \mathrm{kpc})$ scale of 93 local $(0.01<z<0.02)$ massive $\left(M_{*}>10^{10.5} M_{\odot}\right)$ spiral galaxies. To derive spatially-resolved SFR and stellar mass, we perform so-called pixelto-pixel SED fitting, which fits an observed spatially-resolved multiband SED with a library of model SEDs using Bayesian statistics approach. We use 2 bands ( $F U V$ and $N U V)$ and 5 bands $(u, g, r, i$, and $z)$ imaging data from Galaxy Evolution Explorer (GALEX) and Sloan Digital Sky Survey (SDSS), respectively. We find a tight nearly linear relation between the local surface density of SFR $\left(\Sigma_{\mathrm{SFR}}\right)$ and stellar mass $\left(\Sigma_{*}\right)$ which has flattening in high $\Sigma_{*}$. The near linear relation between $\Sigma_{*}$ and $\Sigma_{\mathrm{SFR}}$ suggests constant sSFR throughout the galaxies, and the scatter of the relation is directly related to that of sSFR. Therefore, we analyse the variation of sSFR in various scales. More massive galaxies on average have lower sSFR throughout them than less massive galaxies. We also find that barred galaxies have lower sSFR in a core region than non-barred galaxies. However, in the outside region, sSFR of barred and non-barred galaxies are similar and lead to the similar total sSFR.
\end{abstract}

Key words: galaxies: formation - galaxies: evolution - galaxies: fundamental parameters

\section{INTRODUCTION}

It is already known for decades that among galaxies at narrow redshift range, there is a tight and nearly-linear relation between their $M_{*}$ and star formation rate (SFR), which is called star formation main sequence (SFMS)(e.g., Brinchmann et al. 2004; Elbaz et al. 2007; Noeske et al. 2007; Salim et al. 2007; Whitaker et al. 2012; Speagle et al. 2014; Renzini \& Peng 2015). This relation means that current star formation rate and integrated past star formation rate are tracing each other tightly. Thanks to increasing number of wide-field and multiwavelength surveys, we can examine the relation with a large number of galaxies in a wide redshift range.

As galaxies evolve with redshift, global SFMS relation also evolves with redshift in term of its slope and normalization. The normalization shows a factor of $\sim 2$ dex decrease from high redshift $(z \sim 6)$ to low redshift $(z \sim 0)$, corresponds to decreasing specific SFR (sSFR) with cosmic time. On the other hand, its scatter seems to be independent of redshift with $\sigma$ of $\sim 0.3$ dex (Whitaker et al. 2012; Speagle et al. 2014). The constant small scatter of global SFMS relation

^ E-mail: abdurrouf@astr.tohoku.ac.jp may indicate that star formation inside galaxies is mainly governed by secular process rather than stochastic-merger process (Noeske et al. 2007).

Recently, several investigation on local $\sim 1 \mathrm{kpc}$ scale of star formation found that surface density of SFR $\left(\Sigma_{\mathrm{SFR}}\right)$ and stellar mass $\left(\Sigma_{*}\right)$ are correlated in the form similar as those in the global scale SFMS (Wuyts et al. 2013 (W13); Magdis et al. 2016 (M16); Cano-Díaz et al. 2016 (C16); Maragkoudakis et al. 2017 (M17)). These relations, which are called spatially-resolved SFMSs, are examined by comparing $\Sigma_{*}$ and $\Sigma_{\mathrm{SFR}}$ of sub-galactic regions of local $z \sim 0$ (C16 and M17) and $z \sim 1$ (W13 and M16) star-forming galaxies. Spatiallyresolved SFMS provides the detailed information on the relation between the local star-formation mechanism and the global properties of galaxies.

Spatially-resolved SFMS relations reported in previous research papers differ in terms of slope and normalization. C16 found slope of 0.72 and normalization of -7.95 , while other three papers reported steeper slope close to unity with normalization of $\sim-8.3$ (W13 and M16) and -9.01 (M17). W13 and M16 seem to agree each other, while C16 and M17 result in different spatially-resolved SFMS. Similar to the global scale SFMS relations available in the literature, whose different slope, normalizations, and scatters (see 
Speagle et al. (2014) for the compilation) are mainly caused by the use of different SFR indicators, initial mass function (IMF), stellar population synthesis model, and sample selection. The differences in the spatially-resolved SFMS relations are thought to be caused by differences in methodology (spatially-resolved spectroscopy in C16 and mid-infrared photometry in M17). Therefore, a unique method which can be applied to a large number of galaxies from low to high redshifts is needed to study spatially-resolved SFMS relation and its evolution with redshift.

This work aims to investigate the local sub-galactic $(\sim 1 \mathrm{kpc})$ scale relation between $\Sigma_{*}$ and $\Sigma_{\mathrm{SFR}}$ using so-called pixel-to-pixel SED fitting, which fits spatially-resolved 7 bands ( $F U V, N U V, u, g, r, i$, and $z$ from GALEX and SDSS) photometric SED with a library of model SEDs through Bayesian statistics approach. An advantage of this method is its applicability to various redshifts with nearly consistent manner. In subsequent papers, we will apply the same methodology, spatially-resolved rest-frame $F U V$-NIR SED fitting, to the galaxies at $z \sim 1$ to examine the evolution of the spatially-resolved SFMS relation.

The structure of this paper is as follows. In Section 2 we explain the data sample. Section 3 presents details of the methodology. Result and discussion are presented in Section 4 and Section 5, respectively. Finally, we summarize our results in Section 6. Throughout this paper, cosmological parameters of $\Omega_{m}=0.3, \Omega_{\Lambda}=0.7$, and $H_{0}=70 \mathrm{kms}^{-1} \mathrm{Mpc}^{-1}$ are used. $M_{*}$ will be used to represent total stellar masses of galaxies, while $m_{*}$ will represent the total stellar mass within a sub-galactic region.

\section{DATA SAMPLE}

We examine the relation between $\Sigma_{*}$ and $\Sigma_{\text {SFR }}$ in $1-2 \mathrm{kpc}$ scale, by estimating spatially resolved $m_{*}$ and SFR of massive face-on spiral galaxies in the local universe. In order to estimate $\Sigma_{*}$ and $\Sigma_{\mathrm{SFR}}$, we use $2(F U V$ and $N U V)$ and 5 bands $(u, g, r, i$, and $z$ ) imaging data from GALEX (Morrissey et al. 2007) and SDSS DR10 (Ahn et al. 2014), respectively. The 7 bands span from far-ultraviolet to near-infrared $(1350 \AA \leq \lambda \leq 10000 \AA)$. Thanks to the wide wavelength coverage, the degeneracy between age and dust extinction (existing in the stellar population synthesis modelings) can be broken; the dust extinction can be constrained by $F U V$ $N U V$ colour, while age can be constrained by $u-g$ colour

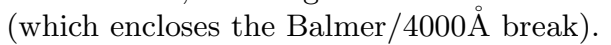

In this analysis, we do not use MIR/FIR imaging dataset of nearby galaxies (e.g., The Spitzer Infrared Nearby Galaxies Survey (SINGS); Kennicutt et al. (2003), Key Insights on Nearby Galaxies: a Far-Infrared Survey with Herschel (KINGFISH); Kennicutt et al. (2011)), because the numbers of massive galaxies in the above MIR/FIR imaging datasets are limited; for example, there are only 9 galaxies more massive than $M_{*}=10^{10.5} M_{\odot}$ in the KINGFISH sample (Kennicutt et al. 2011). Furthermore, in a subsequent paper, we will apply the same methodology, spatially-resolved restframe $F U V$-NIR SED fitting, to the galaxies at $z \sim 1$ to examine the cosmological evolution of the $\Sigma_{*}$ and $\Sigma_{\mathrm{SFR}}$, and imaging dataset with $1 \mathrm{kpc}$ scale resolution is not available in the MIR/FIR wavelength for the galaxies at $z \sim 1$.

The sample galaxies are selected from MPA-JHU (Max
Planck Institute for Astrophysics-Johns Hopkins University) galaxy catalog ${ }^{1}$ (Tremonti et al. 2004) based on their redshifts and $M_{*}$. In the catalog, $M_{*}$ is calculated following a method used in Kauffmann et al. (2003) and Salim et al. (2007), and SFR is calculated based on emission line indices following Brinchmann et al. (2004). We apply the following criteria to the MPA-JHU galaxies that have GALEX imaging data; (1) redshift range of $0.01<z<0.02$, (2) $M_{*}$ more than $10^{10.5} M_{\odot}$, (3) spiral morphology, and (4) face-on disc with ellipticity less than 0.6 or $b / a>0.4$.

The redshift range of the sample is determined, in order to achieve the spatial resolution less than $1 \mathrm{kpc}$ with the GALEX image, which has a FWHM of $5.3^{\prime \prime}$ in the NUV band. Because we focus on the massive galaxies, the $M_{*}$ limit is set. We limit our analysis to spiral galaxies with face-on configuration in order to minimize the effect of dust extinction in highly inclined galaxies. We check the morphological classification of the galaxies selected from the criteria (1) and (2) in Galaxyzoo ${ }^{2}$ project (Lintott et al. 2008), and also inspect the SDSS three colour composite image with $g, r$, and $i$ bands by eye. Ellipticities of the selected spiral galaxies are calculated by averaging $r$-band elliptical-isophotes outside of the effective radius as described in the construction of radial profiles (see Section 4.2). In addition to the above 4 selection criteria, we remove galaxies that are not covered in the GALEX imaging surveys, and only consider galaxies that have more than 4 bins of pixels with $\mathrm{S} / \mathrm{N}$ ratio more than 10 in all of the 7 bands (see Section 3.2 for the binning method). In total 93 galaxies are selected with the above criteria. Figure 1 shows the distribution of $M_{*}$ and SFR of the selected galaxies, which are marked with blue open circles and green open squares for non-barred and barred galaxies, respectively, along with all galaxies at $0.01<z<0.02$ in the MPA-JHU galaxy catalog plotted with black and grey points.

In the later analysis, we consider the existence of bar structure in each spiral galaxy. For the classification of barred and non-barred galaxies, we follow the NASA/IPAC Extragalactic Database (NED) ${ }^{3}$, where morphological classification of all selected galaxies are available. Finally, the detailed morphological classification of the sample is as follows; the non-barred spiral sample contains 4 S0s, 13 spirals earlier type than $\mathrm{Sb}$, and 34 spirals later type than $\mathrm{Sb}$, and the barred spiral sample has 1 SB0, 15 spirals earlier than $\mathrm{SBb}$, and 26 spirals later than $\mathrm{SBb}$. All of the galaxies are earlier type than Scd, and there are no irregular galaxies.

The limiting magnitudes of the SDSS images for stellar objects are 22.3, 23.3, 23.1, 22.3, and $20.8 \mathrm{mag}$ in the $u, g, r, i$, and $z$ filters, respectively (York et al. 2000). The depth and limiting magnitudes of GALEX images depend on the surveys; All Sky Survey (AIS) has exposure time of about $100 \mathrm{sec}$ and limiting magnitudes $(5 \sigma)$ of $19.9 \mathrm{mag}$ in $F U V$ and $20.8 \mathrm{mag}$ in $N U V$, Medium Imaging Survey (MIS) has typical exposure time of $1500 \mathrm{sec}$ and limiting magnitudes $(5 \sigma)$ of $22.6 \mathrm{mag}$ in $F U V$, and $22.7 \mathrm{mag}$ in $N U V$, and Deep Imaging Survey (DIS) has typical exposure time of $30000 \mathrm{sec}$ and limiting magnitudes $(5 \sigma)$ of $24.8 \mathrm{mag}$ in $F U V$

\footnotetext{
1 http://www.mpa-garching.mpg.de/SDSS/DR7/

2 www.galaxyzoo.org

3 https://ned.ipac.caltech.edu/
} 


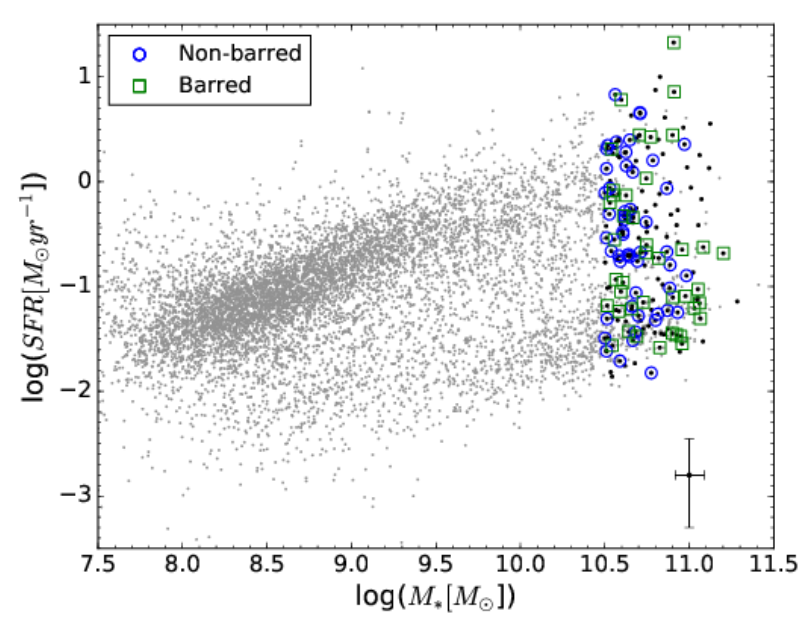

Figure 1. SFR vs. $M_{*}$ of galaxies at $0.01<z<0.02$. Grey dots represent all galaxies in the MPA-JHU galaxy catalog. Large black points represent spiral galaxies with $M_{*}>10^{10.5} M_{\odot}$, while the selected galaxies for this study are marked with blue open circles and green open squares for non-barred and barred galaxies, respectively. Cross symbol in lower left side indicates average uncertainties of $M_{*}$ and SFR.

and $24.4 \mathrm{mag}$ in $N U V$ (Morrissey et al. 2007). SDSS and GALEX images have a spatial sampling of $0.4^{\prime \prime}$ per pixel and $1.5^{\prime \prime}$ per pixel, respectively. The typical PSF sizes are $1.3^{\prime \prime}$ for SDSS images, 5.3" for the GALEX $N U V$ band, and $4.2^{\prime \prime}$ for the GALEX FUV band. The PSF sizes correspond to the physical sizes of $0.3-0.5 \mathrm{kpc}$ (SDSS images), 1-2kpc (GALEX $N U V$ band), and $0.8-1.7 \mathrm{kpc}$ (GALEX $F U V$ band) for galaxies at $z=0.01 \sim 0.02$. Individual pixel corresponds to the spatial scale of $0.3-0.6 \mathrm{kpc}$ in the redshift range.

\section{METHODOLOGY}

We apply a method so called "pixel-to-pixel SED fitting" (e.g., Abraham et al. 1999; Welikala et al. 2008; Zibetti et al. 2009; Wuyts et al. 2012; Sorba \& Sawicki 2015) to derive $\Sigma_{*}$ and $\Sigma_{\text {SFR }}$ in each region of a galaxy. This method can be divided into three main steps; (1) PSF matching, image registration, and binning to obtain 7 bands photometric flux in each bin of a galaxy, (2) construction of model photometric SEDs, and (3) fitting the set of model photometric SEDs to the 7 band photometric flux. In this analysis, SED fitting based on Bayesian inference statistics is used to estimate $m_{*}$ and SFR of a bin. The Probability of each SED model, which has a corresponding $m_{*}$ and SFR, is calculated, then probability distribution functions (PDFs) of $m_{*}$ and SFR are constructed by compounding the probabilities of SED models in a library.

\subsection{PSF Matching and Image Registration}

The images in the $F U V, u, g, r, i$, and $z$ bands are convolved with the kernels to match the PSFs in these bands to that in the $N U V$ band, which has the largest FWHM $\left(5.3^{\prime \prime}\right)$ among the 7 bands. The convolution kernels are calculated using psfmatch command in IRAF ${ }^{4}$, based on the average PSF of each band. The average PSF is determined by combining images of bright and isolated stars in the images. The average PSFs and kernels are calculated with the SDSS sampling (0.4" per pixel), and the convolution is done under the sampling.

Then all the images are registered into the GALEX sampling (1.5" per pixel). Afterward, the images are trimmed within $141 \times 141$ pixels around the galaxy in question. The Fitting region of the galaxy is defined using segmentation maps produced by Sextractor (Bertin \& Arnouts 1996) with a detection threshold of above 1.5 times larger than rms scatter outside of the galaxy applied to all of the 7 bands. The final fitting region of the galaxy is determined by joining all segmentation maps obtained in the 7 bands.

Flux and flux uncertainties in the 7 bands associated with each pixel are calculated by converting from pixel value at each band. All fluxes and flux uncertainties are expressed in erg s $\mathrm{s}^{-1} \mathrm{~cm}^{-2} \AA^{-1}$. The conversion factors from pixel values of $F U V$ and $N U V$ GALEX images (in counts per second) to $F U V$ - and $N U V$-band fluxes are explained in Morrissey et al. (2007). We convert pixel values of SDSS images, which are represented in unit of nanomaggy, to fluxes and flux uncertainties, following the description in the SDSS's website 5 .

\subsection{Pixel Binning}

In order to increase the $\mathrm{S} / \mathrm{N}$ ratio of the resolved $\mathrm{SED}$, we apply binning to the flux and flux uncertainties. We introduce a binning method in which three conditions are considered; (1) closeness by which only connected pixels are binned, (2) similarity of SED's shape, and (3) binned S/N to achieve higher $\mathrm{S} / \mathrm{N}$ in all bands than a threshold value. If we only consider the closeness of pixels' positions for the binning process, it is possible that some pixels may have different SED shapes compared to the other pixels in a bin.

Firstly, the brightest pixel is selected from the trimmed $r$-band image of a galaxy. Then pixels enclosed within a radius of $r=2$ pixel from the brightest pixel are examined for similarity of it's SED shape to that of the brightest pixel. The similarity of the SED shape of a pixel with index of $m$ to that of the brightest pixel with index of $b$ is evaluated with $\chi^{2}$ of

$\chi^{2}=\sum_{i} \frac{\left(f_{m, i}-s_{m b} f_{b, i}\right)^{2}}{\sigma_{m, i}^{2}+\sigma_{b, i}^{2}}$.

$i$ in equation (1) represent image band, and $f_{m, i}$ and $f_{b, i}$ are $i$-th band flux of a pixel $m$ and $b$, respectively. $\sigma_{m, i}$ and $\sigma_{b, i}$ are $i$-th band flux uncertainty of the pixel $m$ and $b . s_{m b}$ corrects scaling difference between the two pixels, and it can

4 IRAF is distributed by the National Optical Astronomy Observatory, which is operated by the Association of Universities for Research in Astronomy, Inc., under cooperative agreement with the National Science Foundation

5 https://www.sdss3.org/dr10/algorithms/magnitudes.php https://data.sdss.org/datamodel/files/BOSS_PHOTOOBJ/ frames/RERUN/RUN/CAMCOL/frame.html 
be calculated using the equation of

$$
s_{m b}=\frac{\sum_{i} \frac{f_{m, i} f_{b, i}}{\sigma_{m, i}^{2}+\sigma_{b, i}^{2}}}{\sum_{i} \frac{f_{b, i}^{2}}{\sigma_{m, i}^{2}+\sigma_{b, i}^{2}}} .
$$

If $\chi^{2}$ is smaller than 30 , the pixel $m$ is binned with the brightest pixel $b$.

After binning all the selected pixels within the radius, the total $\mathrm{S} / \mathrm{N}$ of the bin is checked. If total $\mathrm{S} / \mathrm{N}$ of the bin is above the $\mathrm{S} / \mathrm{N}$ threshold of $\mathrm{S} / \mathrm{N}=10$ in all of the 7 bands, the expansion of the bin is terminated. Otherwise, the radius of the process is increased by $\mathrm{d} r=2$ pixel and the same procedure is applied until the total $\mathrm{S} / \mathrm{N}$ of the bin reach the $\mathrm{S} / \mathrm{N}$ threshold. Once the binning process of the bin is terminated, we look for the brightest pixel in the $r$-band image which has not been included in the previous binning and applies the same procedure again to make the next bin.

The above procedure is applied until no bin can be made with remaining pixels. It is possible that some pixels of a galaxy, especially in the outskirt, are not included in any bin because shapes of their SEDs are different from those of neighbouring bins, and binning them with pixels with similar SEDs cannot reach the required $\mathrm{S} / \mathrm{N}$ threshold. In such case, all of the remaining pixels are binned into one bin, finally.

Figure 2 shows an example of the binning process of one of the sample galaxies (SDSS ObjID 1237652947457998886 or NGC309). The top panel shows its $r$-band image. As shown in the bottom panel, the galaxy has 212 bins shown with different colour encoded by the index of each bin.

\subsection{Construction of a SED library}

The library of the SEDs used for the Bayesian analysis is constructed with the SED models with random parameters. In this analysis, we simply assume a uniform distribution within a certain parameter range, i.e. we do not consider any prior except for the minimum and maximum values.

Firstly, parent model spectra are generated using GALAXEV stellar population synthesis model (Bruzual \& Charlot 2003) with uniform parameter grid. We assume Chabrier (2003) initial mass function (IMF) with mass range between $0.1 M_{\odot}$ and $100 M_{\odot}$ and exponentially declining SFR model $\operatorname{SFR}(t) \propto e^{-t / \tau}$ with $\tau$ of decaying time and $t$ of age. Dust extinction curve of Calzetti et al. (2000) is applied as a screen because the extinction curve reproduces the observed SEDs better than other extinction curves. Figure 3 compares the distribution of the bins of the spatially-resolved photometry of all of the galaxies in the sample (4768 bins) in the $F U V-N U V$ vs. $F U V-u$ and $F U V-N U V$ vs. $N U V-u$ colour-colour plane, and the vectors of the dust reddening effect with Calzetti et al. (2000) and Milky-Way dust (Seaton 1979; Cardelli et al. 1989) extinction laws for a model SED with $Z=0.02, \tau=8.6 \mathrm{Gyr}$, and age $=13.75 \mathrm{Gyr}$. The model's parameters are selected as they are the typical parameters of best-fitting models with zero dust extinction. Milky-Way dust extinction law has the bump feature at around $2175 \AA$, thus reddening effect in $F U V-N U V$ colour is much smaller than that with the Calzetti et al. (2000) extinction law. Because the reddening vector with Calzetti et al. (2000) extinction law broadly follows the observed distribution of the $F U V-N U V$ vs. $F U V-u$ and $F U V-N U V$ vs. $N U V-u$
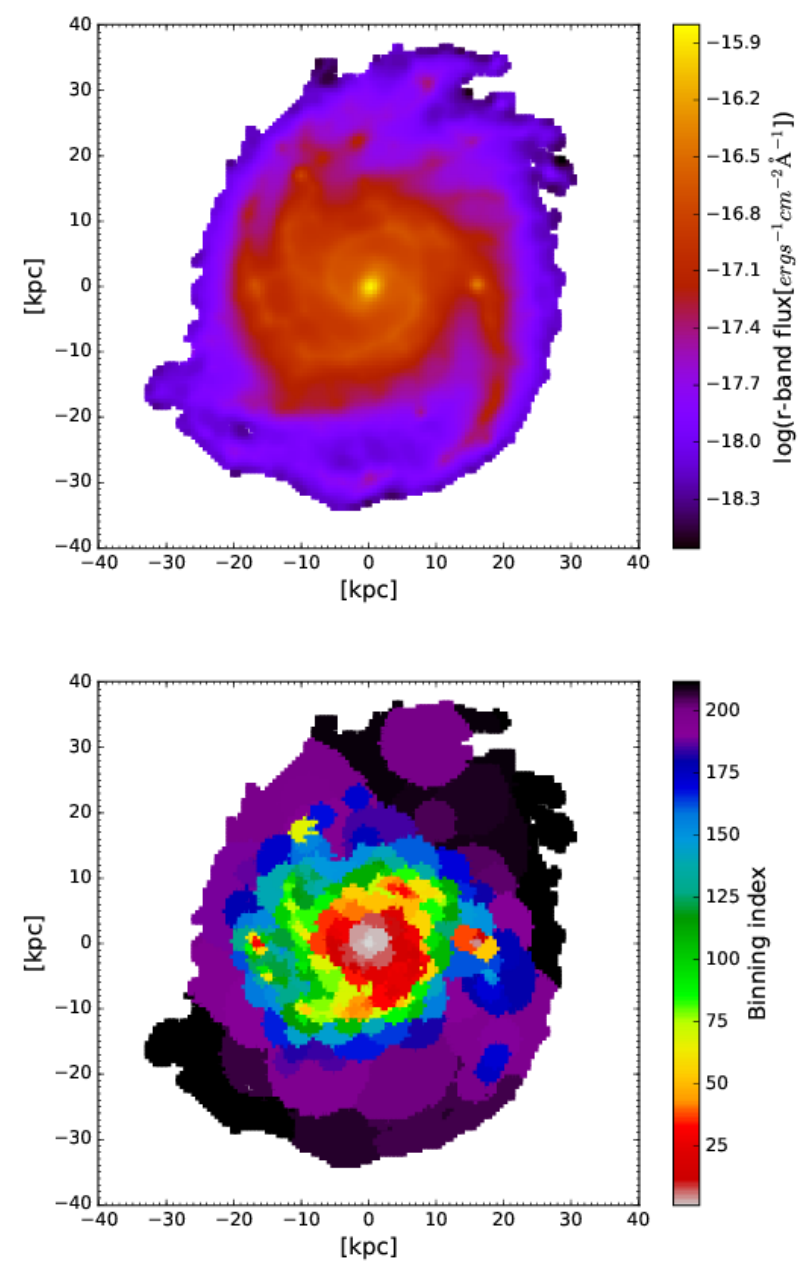

Figure 2. Top panel: $r$-band image of SDSS ObjID 1237652947457998886 (or NGC0309). Bottom panel: the binning result of the galaxy. Different colour encoded with the index of a bin. North is to the top and east is to the left.

colours of the bins better than the Milky-Way dust extinction law, we apply the Calzetti et al. (2000) extinction law in this study (see also Appendix A). model;

We use the following grids of parameters in the parent

(i) 100 values of SFR e-folding time, $\tau$, from 0.1 Gyr to 10 Gyr with $\Delta \tau$ of $0.1 \mathrm{Gyr}$,

(ii) 55 values of age, $t$, from 0.25 Gyr to 13.75 Gyr with $\Delta t$ of $0.25 \mathrm{Gyr}$,

(iii) 13 values of $E(B-V), 0$ (no extinction), 0.05, 0.1, $0.15,0.2,0.25,0.3,0.35,0.4,0.45,0.5,0.55$, and $0.6 \mathrm{mag}$,

(iv) 4 values of metallicities, $\mathrm{Z}: 0.004,0.008,0.02$, and 0.05 .

Then, the model spectrum, which is normalized to the total stellar mass of $1 M_{\odot}$, is converted to the observed flux, $f_{\lambda}(\lambda)$ with the cosmological redshifting and dimming effect. Fluxes of the models in the 7 bands are calculated using

$f_{m, i}=\frac{\int f_{\lambda}(\lambda) \lambda T_{i}(\lambda) d \lambda}{\int \lambda T_{i}(\lambda) d \lambda}$, 
where $T_{i}$ is the $i$ th-band filter transmission function and $f_{m, i}$ is the model's observed-frame flux density through that filter. The $\lambda$ factor in both of the numerator and denominator accounts for the fact that the observations are done with photon counting detectors and the number of photons per unit flux are proportional to the wavelength (Sawicki 2012).

We construct 300,000 photometry models with a random set of parameters $(\tau, t, E(B-V)$, and $Z)$ by interpolating the 286,000 parent model spectra. The sets of parameters are randomly selected in the linear scale within the same ranges as those of the parent models. For each set of parameters, 7 band fluxes and stellar mass are calculated by interpolating at first in 3 dimensional space of $(E(B-V), t$, and $\tau$ ) using tricubic interpolation for each metalicity, then in 1-dimensional space of $Z$ with cubic spline interpolation.

\section{4 $m_{*}$ and SFR from the SED fitting with Bayesian statistics}

$m_{*}$ and SFR of each bin of a galaxy are estimated with the Bayesian statistics. At first, in each bin probability distribution functions (PDFs) of SFR and $m_{*}$ are constructed, and then the posterior means of SFR and $m_{*}$ are calculated. Because the model SEDs are normalized with a stellar mass of $1 M_{\odot}$, the estimated normalization corresponds to the $m_{*}$. Instantaneous SFR is derived with

$S F R=\frac{m_{*}}{1-\exp (-t / \tau)} \times \frac{\exp (-t / \tau)}{\tau}$

where first multiplication term in the right-hand side serve as scaling between integrated mass and instantaneous SFR. The probability functions are constructed based on the $\chi^{2}$ statistics. The $\chi^{2}$ value of the $m$-th model for the SED of a $d$-th bin is defined with

$\chi_{m}^{2}=\sum_{i}\left(\frac{f_{d, i}-s f_{m, i}}{\sigma_{i}}\right)^{2}$,

where $s$ represents the scaling of the model SED. The scaling factor $s$ is randomly drawn between $0.1 \times s_{\text {least }}$ to $10 \times s_{\text {least }}$, where $s_{\text {least }}$ is the scaling of the minimum $\chi^{2}$ solution, that is

$s_{\text {least }}=\frac{\sum_{i} \frac{f_{d, i} f_{m, i}}{\sigma_{i}^{2}}}{\sum_{i} \frac{f_{m, i}^{2}}{\sigma_{i}^{2}}}$.

For each model SED, 100 random $s$ are generated and $\chi^{2}$ of model SED with each $s$ is calculated.

The posterior mean of the SFR and $m_{*}$ of a bin are calculated by

$\bar{\theta}=\int \theta P(\theta \mid X) d \theta$,

with $\theta$ stands for SFR or $m_{*}$, and $P(\theta \mid X)$ is the probability of a model with a value of $\theta$ given an observational data set, $X$.

We use a PDF in the form of the Student's tdistribution, which gives higher probability to a model with large $\chi^{2}$ compared to the Gaussian distribution $(P(\theta \mid X) \propto$ $\left.\exp \left(-\chi^{2} / 2\right)\right)$, which is commonly used in the Bayesian SED fittings (e.g., Kauffmann et al. 2003; Salim et al. 2007; da Cunha et al. 2008; Walcher et al. 2011; Han \& Han 2014). We assume the degree of freedom of the Student's t-distribution, $v$, as 3 , because that distribution gives the consistent sSFR estimate as tested with mock SEDs and also gives the best SFR estimate as compared to $24 \mu \mathrm{m}$ observations (see Appendix A). The normalized PDF has a form as

$P(\theta \mid X)=\frac{W_{\theta m}}{\sum_{m=1}^{N} W_{\theta m}}$

with

$W_{\theta m}=\frac{\Gamma\left(\frac{v+1}{2}\right)}{\sqrt{v \pi} \Gamma\left(\frac{v}{2}\right)}\left(1+\frac{\left(\chi_{m}^{2}\right)^{2}}{v}\right)^{-\frac{v+1}{2}}$

with $W_{\theta m}$ represents the weight of the $m$-th SED model which has $\theta$ (SFR or $m_{*}$ ), and $N$ is the total number of the SED models, which is $100 \times 300,000$.

Once the posterior means of the SFR and $m_{*}$ of each bin are calculated, those values are divided into the pixels which belong to the bin. We assume that the SFR and $m_{*}$ of a pixel are proportional to its $F U V$ and $z$ band flux, respectively. We also calculate uncertainties of SFR and $m_{*}$ estimates by calculating the standard deviation with the PDFs of SFR and $m_{*}$.

\section{RESULTS}

\subsection{Spatially-resolved Star Formation Main Sequence}

Figure 4 shows pixel-to-pixel SED fitting result of a galaxy in the sample (SDSS ObjID 1237652947457998886 or NGC0309, whose pixel binning result is shown in Figure 2). The SFR surface density, $\Sigma_{\mathrm{SFR}}$, map clearly traces spiral arm pattern and some knots which enhanced star formation activities are associated, while the $m_{*}$ surface density, $\Sigma_{*}$, map shows smoother distribution.

Figure 5 shows integrated $M_{*}$ vs. SFR of the 93 galaxies in the sample. The black line represents integrated star formation main sequence (SFMS) of Speagle et al. (2014) (calculated at the median redshift of the sample, $z=0.0165$ ) with \pm 0.3 dex scatter shown by grey-shaded region. This integrated SFMS is obtained by calculating the total stellar mass, $M_{*}$, and SFR of each galaxy by summing up those values within the fitting region. The uncertainties associated with $M_{*}$ and SFR are calculated by propagation error of summation. The points are distributed around the black line.

To examine the relation between $\Sigma_{*}$ and $\Sigma_{\mathrm{SFR}}$ in $\leq 1-2$ kpc scale, the $\Sigma_{*}$ and $\Sigma_{\text {SFR }}$ of all 375,215 pixels of the sample galaxies are plotted in the left panel of Figure 6 . The figure shows scatter of the pixels and contour colour-coded with the number in each $0.1 \times 0.1 \mathrm{dex}$ bin. As shown by the contour with a high number density, there is a tight relation between $\Sigma_{*}$ and $\Sigma_{\mathrm{SFR}}$. Fitting the data with a linear relation with the form of

$\log \Sigma_{\mathrm{SFR}}=\alpha \log \Sigma_{*}+\beta$.

through least-square fitting method, resulted in the bestfitting relation, which is shown with red dashed line and has a slope $(\alpha)$ of 0.33 and zero point $(\beta)$ of -5.23 with a scatter $(\sigma)$ of 0.70 dex. The best-fitting relation looks largely 

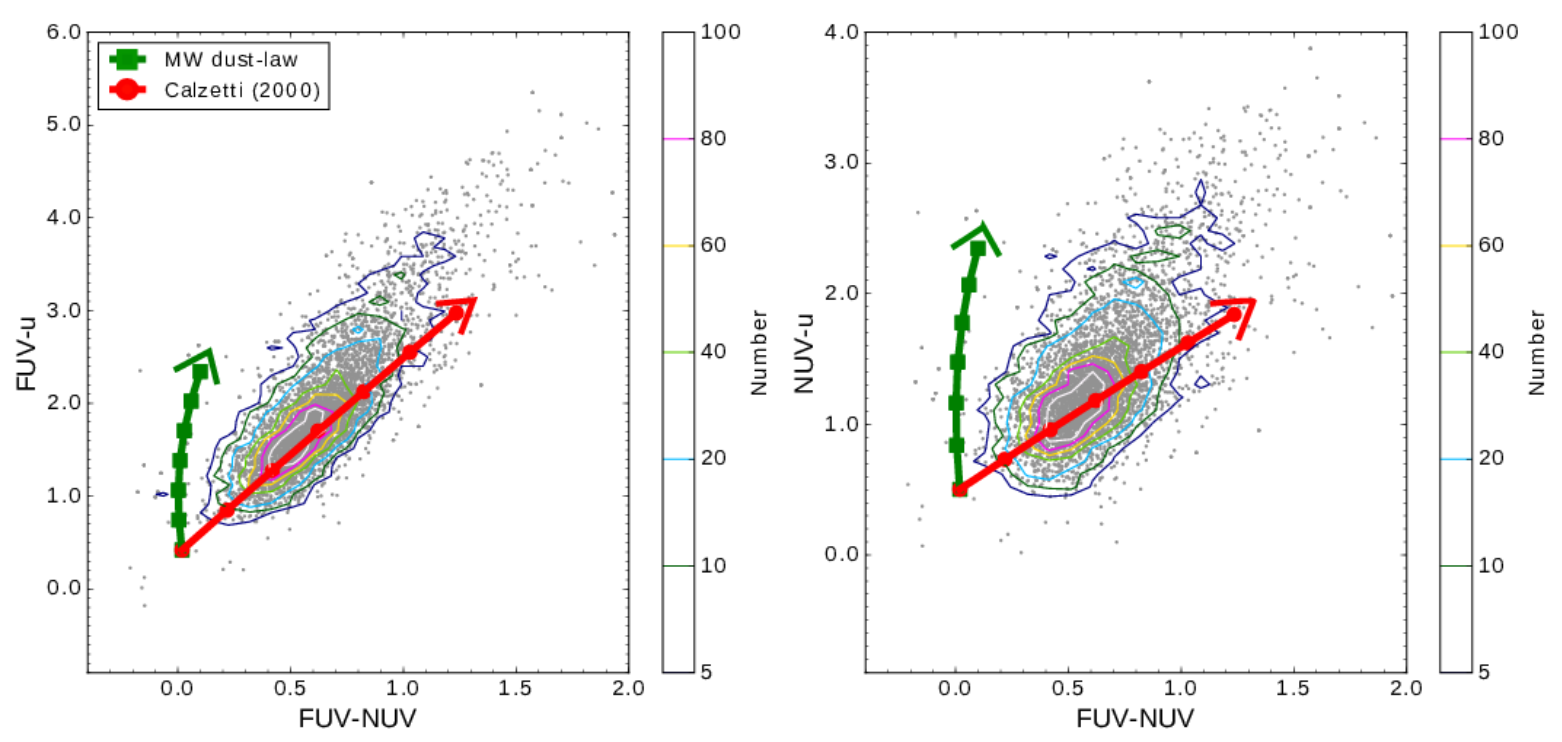

Figure 3. Comparison between the dust extinction tracks of (left panel: $F U V-N U V$ vs. $F U V-u$, right panel: $F U V-N U V$ vs. $N U V-u$ ) Calzetti (2000) and Milky Way dust extinction laws for a model SED with $Z=0.02, \tau=8.6 \mathrm{Gyr}$, and age $=13.75 \mathrm{Gyr}$, in the colour-colour diagrams along with the distribution of the photometries of the bins in the galaxies. The circles and squares mark increasing colour excess $E(B-V)$ from 0 to 0.6 with step of 0.1 .

deviate from overall data distribution shown by contours which is caused by large scatter of high-mass pixels with low sSFR. Therefore, a more objective method is needed to calculate the slope and zero point of the main-sequence. Renzini \& Peng (2015) proposed an objective way to define the main-sequence by calculating mode at "fixed $M_{*}$ " which corresponds to the peak of SFR distribution at fixed $M_{*}$. Although the method is developed for the integrated SFMS, but it is still relevant for the spatially-resolved SFMS. Black circles in the left panel of Figure 6 show mode of $\Sigma_{\text {SFR }}$ distribution for $\Sigma_{*}$ bin with 0.3 dex width. Errorbars represent standard deviation from the mode and calculated separately above and below the mode value. The mode values as a function of $\Sigma_{*}$ seem to well follow the distribution of the data shown by the contours. Steepening (flattening) in lower (higher) than $\log \Sigma_{*} \sim 7.5$ is clearly shown by the mode values, while one should note that flattening in two lowest mode values is affected by the detection limit.

In the right panel of Figure 6, sSFR, which is a ratio between $\Sigma_{\mathrm{SFR}}$ and $\Sigma_{*}$, is plotted as a function of $\Sigma_{*}$. The black circles show the mode of SSFR distribution for a $\Sigma_{*}$ bin with 0.3 dex width. The errorbar is calculated in the same way as those in the left panel. The general trend shows decline of sSFR with $\Sigma_{*}$ with a flat profile in the $\Sigma_{*}$ range, $10^{6.15} M_{\odot} \mathrm{kpc}^{-2} \lesssim \Sigma_{*} \lesssim 10^{7.65} M_{\odot} \mathrm{kpc}^{-2}$, which correspond to linear increase in the same $\Sigma_{*}$ range in the left panel. Applying linear function fitting to the four mode values enclosed within the red contour, resulted in steeper slope $(\alpha), 1.00$ with zero point $(\beta)$ of -9.58 , which represented by the black solid line. Firstly, the zero point $(\beta)$ is determined by the average $\log (\mathrm{sSFR})$ of the four values, then the best slope $(\alpha)$ is determined by a fitting with equation (10). The black solid line well represents the distribution of the data shown by the contours especially in the peak of the distribution.

It is possible that this result has implication on the nearly proportionality relation between an integrated SFR and a total stellar mass of a disc, $M_{* \text {,disc }}$, without a bulge component, of the local star-forming galaxies observed by Abramson et al. (2014).

Recently reported spatially-resolved SFMS in the literature (W13, M16, C16, and M17) are all defined with star-forming galaxy sample. Here, our sample contains some galaxies lie far below the main-sequence (see Figure 5) which seem to be quenched galaxies. These galaxies have lower spatially-resolved sSFR than those galaxies in the main-sequence (MS). This is one of the contributors to the large scatter of the spatially-resolved SFMS derived in this work. Therefore, here we separate galaxies sample into three groups based on their position relative to the SFMS relation of Speagle et al. (2014): above-MS (above +0.3 dex, contains 50 galaxies), on-MS (within \pm 0.3 dex, contains 34 galaxies), and below-MS (below -0.3 dex, contains 9 galaxies). Figure 7 shows spatially-resolved SFMS of galaxies above-MS (top-left, contains 218593 pixels), on-MS (top-right, contains 120161 pixels), and below-MS (bottom-left, contains 36461 pixels). Spatially-resolved SFMS is clearly shown in the first group (above-MS) with steepening (flattening) in low (high) $\Sigma_{*}$ similar as those in the left panel of Figure 6 (for all galaxies). The best-fitting linear function (represented by the black solid line) to the linear increasing part of the mode values (consist of four mode values), calculated with the same method as for Figure 6, has a slope of 0.99 and zero point of -9.59 . Furthermore, linear function fitting to all scattered data in this group resulted in lower scatter, $(\sigma)$ of 0.55 , than those for all galaxies. The black dashed line in each panel is the same as the black solid line in the left panel of Figure 6. In the bottom right panel, mode profiles of the three groups are compared, which shows systematically different normalization (which corresponds to the difference in sSFR) between the three groups. There is no clear trend of spatially-resolved SFMS in the quenched galaxies as shown in the bottom-left panel. 

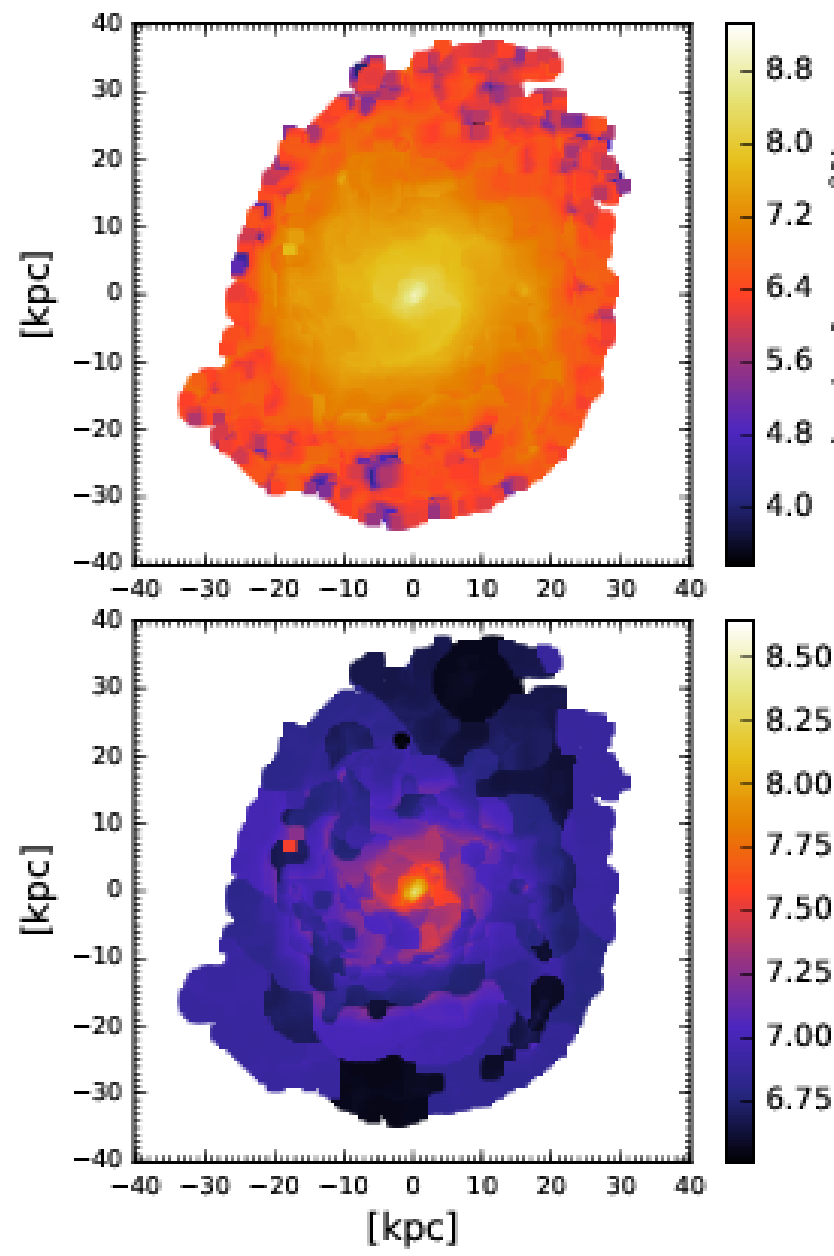

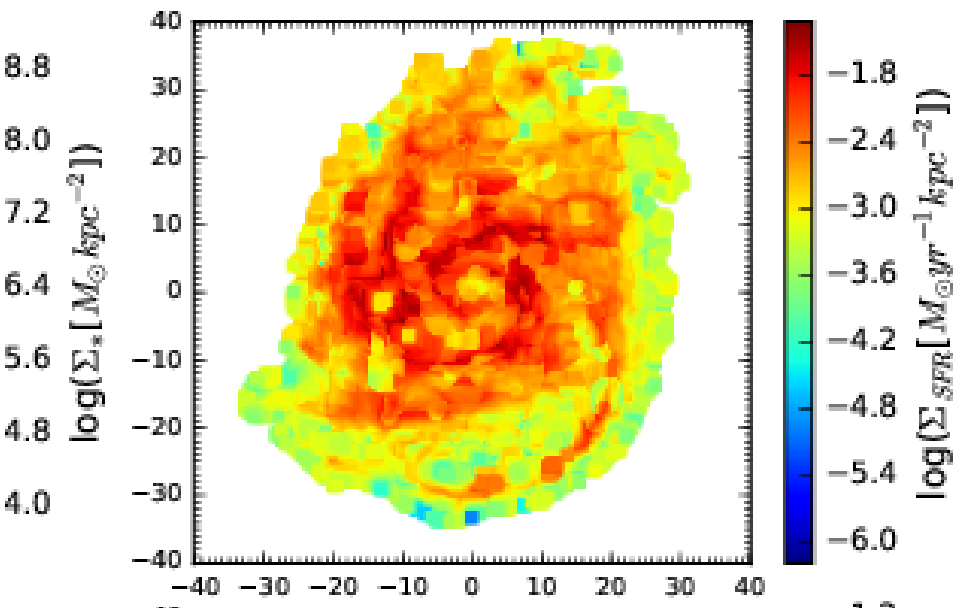

8.250

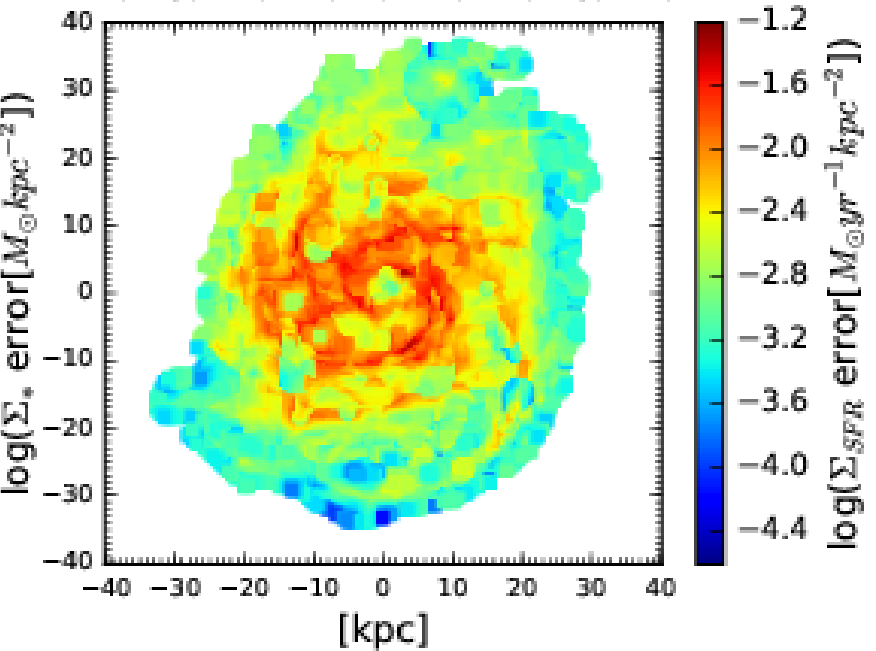

Figure 4. An example of the pixel-to-pixel SED fitting of a galaxy (SDSS ObjID 1237652947457998886 or NGC0309). Top-left panel: $m_{*}$ surface density map, top-right panel: SFR surface density map, bottom-left panel: uncertainty of $m_{*}$ surface density map, and bottom-right panel: uncertainty of the SFR surface density map. Up and left directions correspond to the north and east respectively.

The systematic deviation from a constant sSFR implies systematically different spatially-resolved SFMS in the location. Therefore, hereafter, we examine the systematic offset of sSFR in various scale. At first, Figure 8 shows sSFR map of the galaxy SDSS ObjID 1237652947457998886 or NGC0309. The spiral arms structure is clearly shown on the map indicating the enhanced sSFR along the spiral arms compare to the underlying disc region. Therefore, on the smallest scale, the spatially-resolved SFMS in the spiral arm regions has higher normalization than that in the underlying disc region.

\subsection{Radial Profiles of $\Sigma_{\mathrm{SFR}}, \Sigma_{*}$, and SSFR}

In order to examine the radial variation of the spatiallyresolved SFMS, we construct the radial profiles of the sSFR. Firstly, radial profiles of $\Sigma_{*}$ and $\Sigma_{\mathrm{SFR}}$ of each galaxy are constructed as a function of the semi-major axis of ellipsoids. The ellipsoids are determined by applying ellipticalisophotes fitting to the $r$-band images of the galaxies using ellipse command in IRAF. Then average ellipticity and po- sition angle are derived using ellipsoids outside of the halfmass radius of a galaxy. The half-mass radius is defined with the length of semi-major axis enclosing half of the total $M_{*}$ calculated with average ellipticity and position angle in the entire radius range. We only consider ellipsoids in the outer region of a galaxy, in order to escape from an effect of an existence of bulge and bar components. The radial profile is sampled in $2 \mathrm{kpc}$ step within $6 \mathrm{kpc}$, and $4 \mathrm{kpc}$ step outside of it. We extrapolate the radial profiles of each galaxy up to $40 \mathrm{kpc}$ by fitting the exponential law outside of $6 \mathrm{kpc}$ as follows,

$\psi(r)=\psi_{0} e^{r / h}$

with $\psi$ stand for $M_{*}$ or SFR. $\psi_{0}$ and $h$ represent zero point and radial scale-length, respectively. Figure 9 shows normalized average radial profiles of $\Sigma_{*}$ and $\Sigma_{\mathrm{SFR}}$ which are constructed by calculating the average of the radial profiles, then normalize the average radial profiles with their central values. The normalized average radial profile of $\Sigma_{\text {SFR }}$ has shallower and more extended profile than the average ra- 


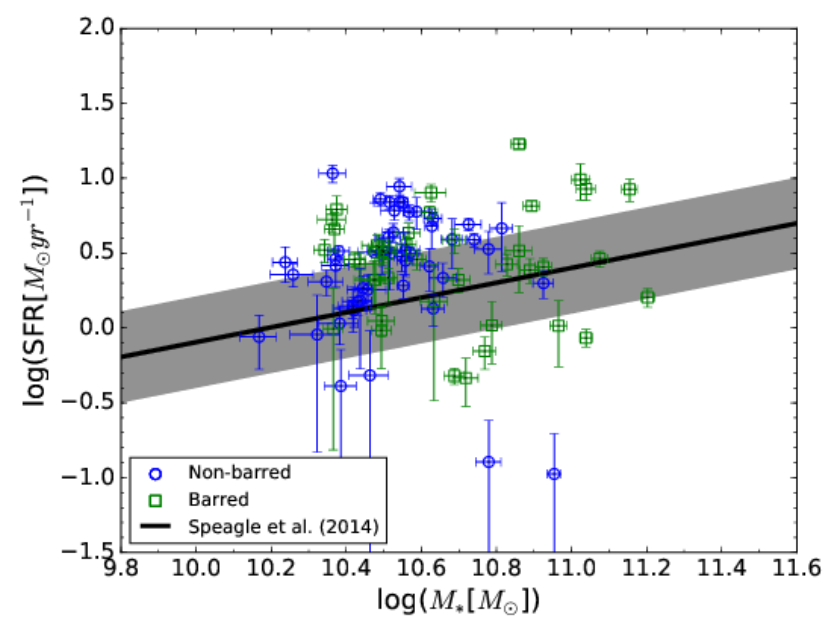

Figure 5. Relation between the integrated $M_{*}$ and SFRs. Blue open circles and green open squares represent non-barred and barred galaxies, respectively. The Black line represents integrated SFMS relation of Speagle et al. (2014). Grey-shaded region around the line represents \pm 0.3 dex scatter.

dial profile of $\Sigma_{*}$ which shows more centrally concentrated distribution.

Utilizing the individual and averaged radial profiles of $\Sigma_{*}$ and $\Sigma_{\mathrm{SFR}}$, we derive profiles of sSFR. Figure 10 shows $\operatorname{sSFR}(r)$ profiles of all of the 93 galaxies. Black cross represent extrapolated part of the profile. The average profile is shown with green filled circles. The average radial profile of sSFR is increasing as increasing radius with stronger increase found around the central region $(r \gtrsim 6 \mathrm{kpc})$. The profile is nearly flat within the radius range of $10 \mathrm{kpc} \leq r \leq$ $22 \mathrm{kpc}$, which agree with the existence of spatially-resolved SFMS (see Section 4.1). The sSFR in the central region has on average 0.9 dex smaller than those in the outer disc region. Such decline is consistent with an existence of a bulge component with an old stellar population and with the recent results with integral field spectroscopy (González Delgado et al. 2016). The Inverse of the sSFR corresponds to the time scale of the star-formation. The average radial profile of the sSFR of the sample show lower than $10^{-10} \mathrm{yr}^{-1}$ in the central $r \lesssim 6 \mathrm{kpc}$ region but higher than $10^{-10} \mathrm{yr}^{-1}$ outside of it $(r>6 \mathrm{kpc})$. This critical sSFR corresponds to the Hubble time.

\section{DISCUSSION}

\subsection{Radial variation of the spatially-resolved SFMS}

In this discussion, we focus on the variation of spatiallyresolved SFMS in large scale, and we evaluate the systematic variation utilizing the radial dependence of the SSFR. In order to quantify the difference in the profiles of sSFR among the galaxies, we derive the ratio between the integrated sSFR inside and outside of the half-mass radius $\left(r_{e, M_{*}}\right)$ same as those defined in Section 4.2. If the ratio becomes larger (smaller) than 1 , we expect sSFR is higher in the inner (outer) region. Figure 11 shows the ratios as a function of the total SFR. As expected from the radial profiles of $\Sigma_{*}$ and $\Sigma_{\mathrm{SFR}}$, most of the galaxies have the ratio smaller than 1 .

By checking the morphologies of the galaxies as a function of the ratio, we found that galaxies that have small ratios are dominated by barred galaxies. In the Figure 11, barred and non-barred galaxies are shown with green squares and blue circles, respectively. The barred galaxies have smaller ratios on average, median values of barred and nonbarred galaxies are -1.08 and -0.78 dex, respectively. The KS-test of the difference in the distributions of the two samples implies that they are different with a significance level of 0.05. Statistical D- and P-values of the KS-test are shown in the histogram of Figure 11. The results indicate that the star-formation of the barred galaxies is more suppressed in the inner region compared to the non-barred galaxies.

In order to further examine the difference in the barred and non-barred galaxies, we divide the galaxies into three regions: "core" defined as region inside $10^{-0.5} r_{e, M_{*}}$, "middle" defined as $10^{-0.5} r_{e, M_{*}}<r \leqslant r_{e, M_{*}}$, and "outside" defined as $r>r_{\boldsymbol{e}, M_{*}}$ following Wuyts et al. (2013). Figure 12 shows the distributions of summed SFRs, $m_{*}$, and sSFRs in the core, middle, and outside regions. As can be seen in the histograms, barred- and non-barred galaxies have similar SFRs each other in the three regions. The $m_{*}$ distribution of barred- and non-barred galaxies are significantly different in the core region, where barred galaxies have higher $m_{*}$ than non-barred galaxies, while the difference in the middle and outside regions are small. sSFR distribution of barredand non-barred galaxies are slightly different in core and middle, where barred-galaxies have lower median than nonbarred galaxies, while in the outer region they have similar value.

Global morphology of a galaxy is affecting the spatiallyresolved SFMS, but limited to the core of a galaxy. In the core region, barred galaxies have lower sSFR than nonbarred galaxies. It has been suggested that a bar structure can quench star formation of galaxies (Cheung et al. 2013; Gavazzi et al. 2015; Zhou et al. 2015; Spinoso et al. 2017). Numerical simulation of a bar formation in a galaxy shows that gas is rapidly transferred toward the central region on the onset of the bar structure, and that the gas transfer triggers a burst of star formation in the central region of the galaxy. As the gas being transferred to the central region, gas within the bar's corotation radius is consumed and SFR within the radius can be suppressed finally (Spinoso et al. 2017).

Interestingly, such suppression of the sSFR is only seen in the core region and there is no difference of $\mathrm{SSFR}$ in the outer region between barred and non-barred galaxies. The total sSFRs of barred and non-barred galaxies do not show a significant difference, and this opposes the arguments that bar structure can quench the star formation activity in the entire region. The similarity of the distributions of sSFR in the middle and outside regions suggests that the spatiallyresolved SFMS does not vary in the outside of the core region, and does not depend on the existence of a bar structure in the central region of a disc. 

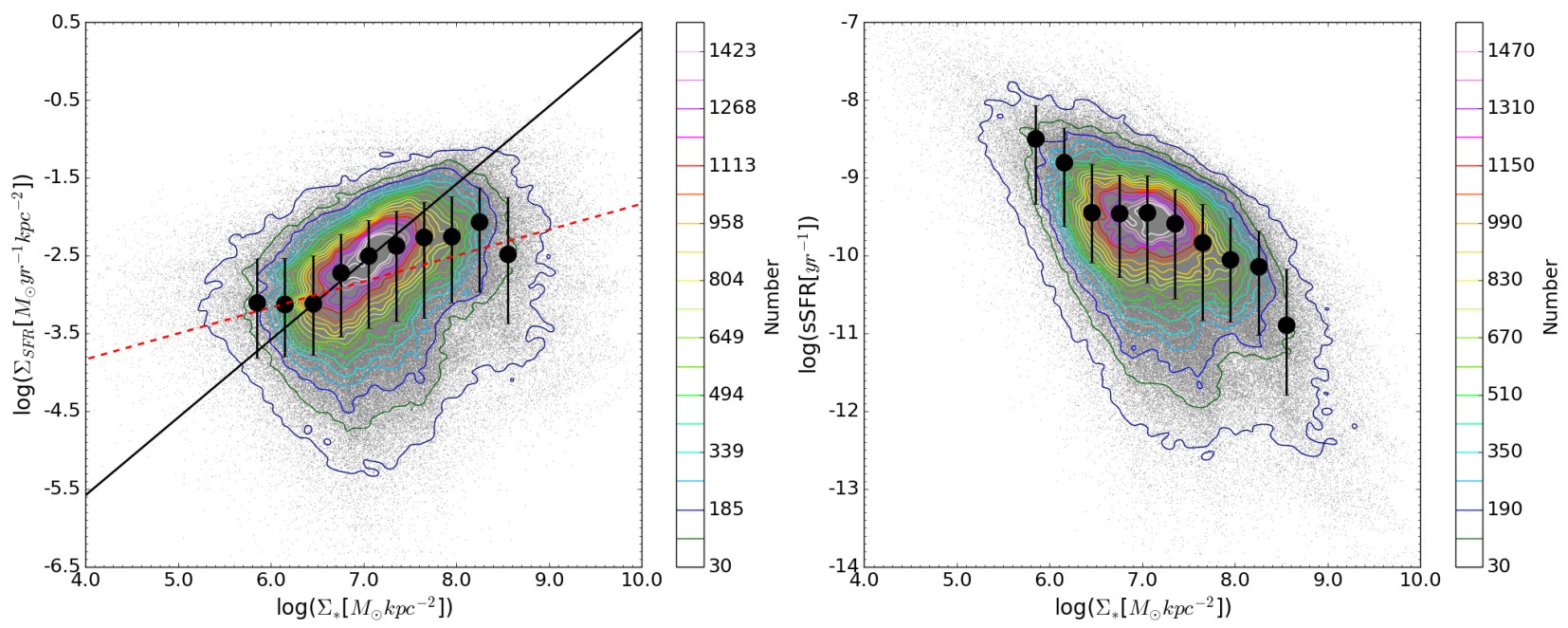

Figure 6. Left panel: Distribution of surface $\boldsymbol{M}_{*}$ and SFR density of the pixels along with contours colour coded by number in each bin. Red dashed line represents linear function fitting to the data. Black circles with errorbars show the mode of $\Sigma_{\text {SFR }}$ distribution for $\Sigma_{*}$ bin with a 0.3 dex width. The Black solid line represents a linear function fitting to the linear increasing part of the mode values. Right panel: Distribution of $\Sigma_{*}$ vs. sSFR. Black circles with errorbars show modes of sSFR distribution for $\Sigma_{*}$ bin with 0.3 dex width.
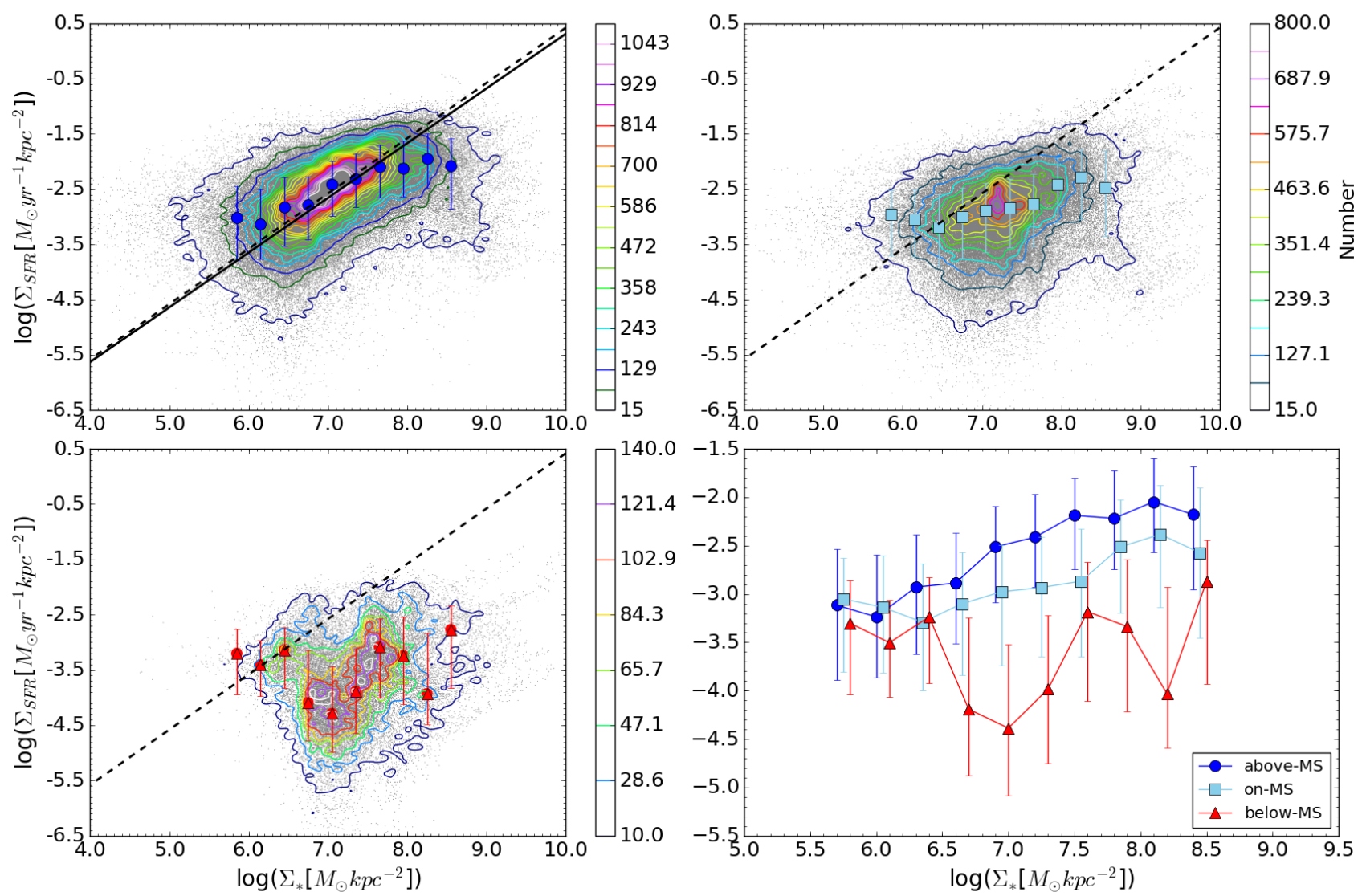

Figure 7. Spatially-resolved SFMS from galaxies above the main-sequence (top-left panel), on the main-sequence (top-right panel), and below the main-sequence (bottom-left panel). Global main-sequence used here is following Speagle et al. (2014) (see Figure 5). Black solid line in the top-left panel represents a linear function fitting to the linear increasing part of the mode values. Black dashed lines in three panels are the same as the black solid line in the left panel of Figure 6. Bottom-right panel: Comparison between the mode profiles of all the three groups. For clarity, blue circles are shifted by 0.05 dex to the left, while red triangles are shifted by 0.05 dex to the right from their actual positions. 


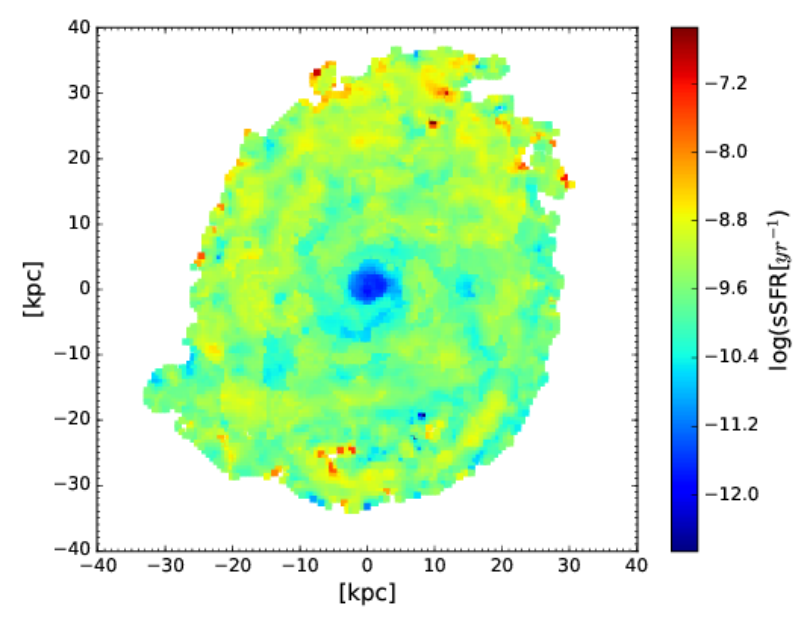

Figure 8. Spatial variation of the sSFR in galaxy SDSS ObjID 1237652947457998886 or NGC0309.

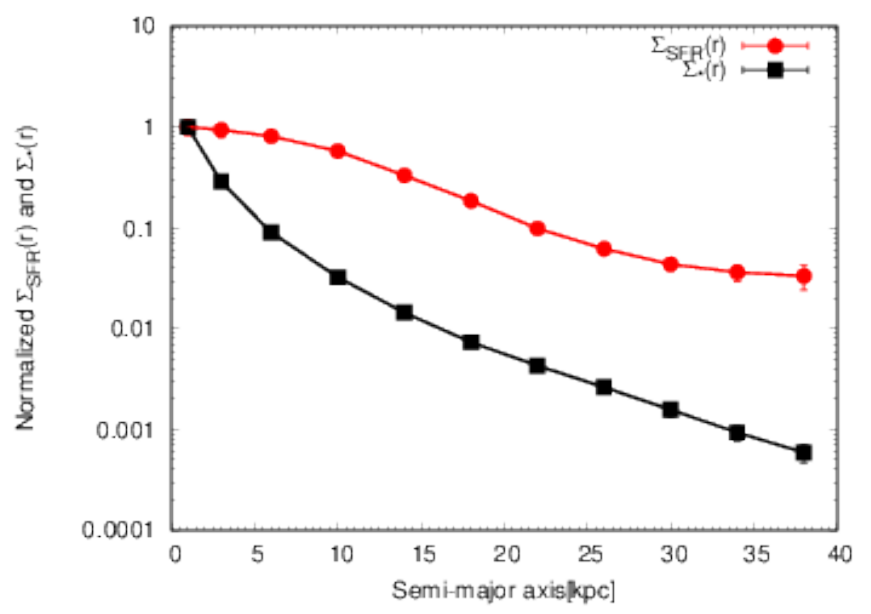

Figure 9. Comparison between normalized average $\Sigma_{*}$ (black filled square) and $\Sigma_{\mathrm{SFR}}$ (red filled circle) radial profiles. The radial profiles are constructed by calculating average of the radial profiles then normalized the average radial profiles by their central values. The errorbars are calculated from standard error of mean.

\subsection{The Dependence of $\operatorname{sSFR}(r)$ Profile on Integrated $M_{*}$}

In order to examine the dependence of spatially-resolved SFMS on the global property of galaxies, we divide the sample into two groups based on their $M_{*}$ at the median $M_{*}$ of $3.5 \times 10^{10} M_{\odot}$. Figure 13 shows the comparison between $\operatorname{sSFR}(r)$ profiles of the high- and low-mass groups. Both of the profiles are increasing as increasing radius and show sharp decline in the central region. These shapes are similar to the average of the entire sample shown in Figure 10. In all radius, the $\operatorname{sSFR}(r)$ of the low-mass group is higher than the high-mass group.

Figure 14 shows comparison between mode values of sSFR in each 0.3 dex bin of $\Sigma_{*}$ for high- and low-mass galaxies. It is shown that high-mass group on average has lower

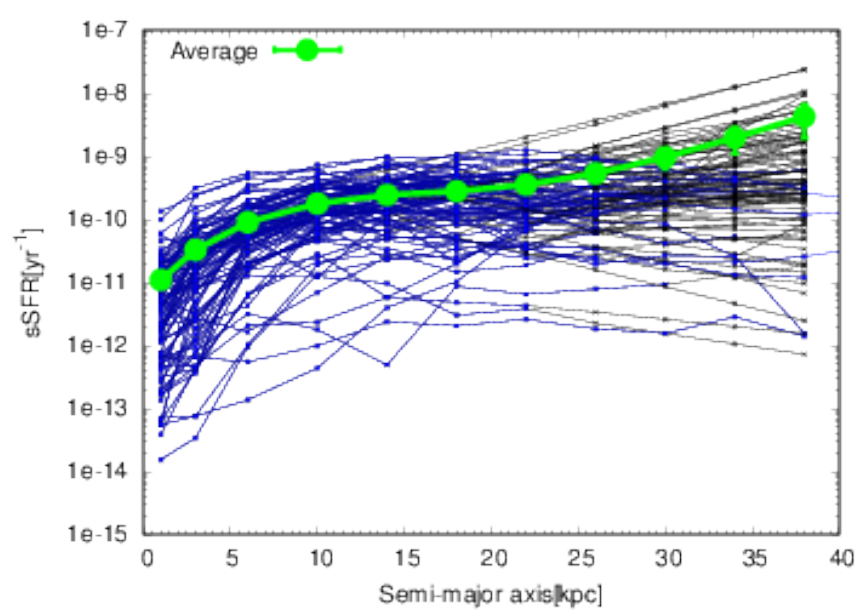

Figure 10. $\operatorname{sSFR}(r)$ profiles of 93 galaxies in the data sample (shown with blue filled square) with extrapolated part of the profile (shown with black cross) and their average (shown with green colour). The error bars are calculated from standard error of mean.

local sSFR than low-mass group for a spatial region with the same $\Sigma_{*}$.

From the integrated SFMS, with a slope value of 1.0, it is known that galaxies with higher masses have smaller total sSFR. Here it is shown that higher mass galaxies have smaller sSFR in all radii, and it is suggested that they have systematically different spatially-resolved SFMS from lessmassive galaxies. Pérez et al. (2013) also show the evidence of systematically smaller sSFR of massive galaxies by constructing their spatially resolved stellar mass assembly rates which are derived from an analysis of their spatially resolved spectra.

\section{SUMMARY}

We examine the relation between local (1-2 kpc-scale) surface density of stellar mass $\left(\Sigma_{*}\right)$ and star formation rate $\left(\Sigma_{\mathrm{SFR}}\right)$ by analysing the $\Sigma_{*}$ and $\Sigma_{\mathrm{SFR}}$ of 93 local massive $\left(M_{*}>10^{10.5} M_{\odot}\right)$ face-on spiral galaxies located at $0.01<z<0.02$. The method so-called pixel-to-pixel SED fitting is used to derive the $\Sigma_{*}$ and $\Sigma_{\mathrm{SFR}}$. We find a tight nearly linear relation between local $\Sigma_{*}$ and $\Sigma_{\text {SFR }}$ which has monotonic increase (with slope nearly unity) in low $\Sigma_{*}$ (corresponds to the disc region) while nearly flat in high $\Sigma_{*}$ (corresponds to the central region). This relation in a local scale is called spatially-resolved star formation main sequence (SFMS). The spatially-resolved SFMS relation seems to break in quenched galaxies.

Analysis on the systematic offset from a constant sSFR in various scale suggested that the scatter in spatiallyresolved SFMS is in part due to the local variation of SSFR, where spiral arm region has enhanced sSFR compared to the underlying disc region, and systematic variation of spatiallyresolved SFMS in larger scale due to global properties, such as $M_{*}$ and the existence of a bar structure. $M_{*}$ has a role in suppressing the local sSFR throughout the galaxy's region in such a way that more massive galaxies tend to have local 


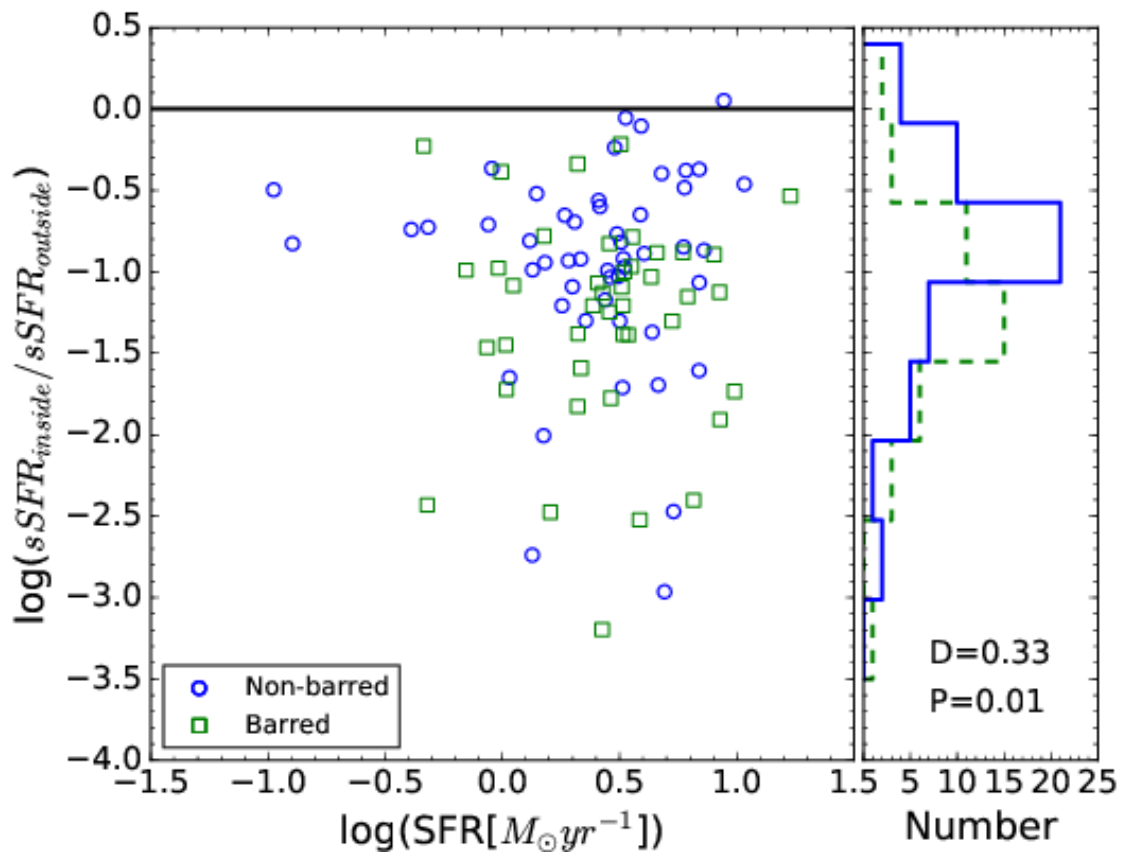

Figure 11. Total SFR vs. logarithmic ratio of $\mathrm{SFR}_{\text {inside }} / \mathrm{SFR}_{\text {outside }}$ of the sample galaxies. Barred and non-barred galaxies are shown with green open squares and blue open circles, respectively. Right panel shows the distribuions of the ratio for barred (green dashed-line) and non-barred (blue solid-line) galaxies.

sSFR lower in all radius than those of less massive galaxies, hence giving a contribution to the scatter in the spatiallyresolved SFMS. Barred galaxies in our sample have systematically lower sSFR in the core region than non-barred galaxies, but their sSFR are similar in the outside regions.

\section{ACKNOWLEDGEMENTS}

This work is based on observations made with the NASA Galaxy Evolution Explorer. GALEX is operated for NASA by the California Institute of Technology under NASA contract NAS5-98034. This work has made use of SDSS data. Funding for SDSS-III has been provided by the Alfred P. Sloan Foundation, the Participating Institutions, the National Science Foundation, and the U.S. Department of Energy Office of Science. The SDSS-III web site is http://www.sdss3.org/. SDSS-III is managed by the Astrophysical Research Consortium for the Participating Institutions of the SDSS-III Collaboration including the University of Arizona, the Brazilian Participation Group, Brookhaven National Laboratory, Carnegie Mellon University, University of Florida, the French Participation Group, the German Participation Group, Harvard University, the Instituto de Astrofisica de Canarias, the Michigan State/Notre Dame/JINA Participation Group, Johns Hopkins University, Lawrence Berkeley National Laboratory, Max Planck Institute for Astrophysics, Max Planck Institute for Extraterrestrial Physics, New Mexico State University, New York University, Ohio State University, Pennsylvania State University, University of Portsmouth, Princeton University, the Spanish Participation Group, University of Tokyo, Uni- versity of Utah, Vanderbilt University, University of Virginia, University of Washington, and Yale University.

\section{REFERENCES}

Abraham R. G., Ellis R. S., Fabian A. C., Tanvir N. R., Glazebrook K., 1999, MNRAS, 303, 641

Abramson L. E., Kelson D. D., Dressler A., Poggianti B., Gladders M. D., Oemler Jr. A., Vulcani B., 2014, ApJ, 785, L36

Ahn C. P., et al., 2014, ApJS, 211, 17

Bertin E., Arnouts S., 1996, A\&AS, 117, 393

Brinchmann J., Charlot S., White S. D. M., Tremonti C., Kauffmann G., Heckman T., Brinkmann J., 2004, MNRAS, 351, 1151

Bruzual G., Charlot S., 2003, MNRAS, 344, 1000

Calzetti D., Armus L., Bohlin R. C., Kinney A. L., Koornneef J., Storchi-Bergmann T., 2000, ApJ, 533, 682

Cano-Díaz M., et al., 2016, ApJ, 821, L26

Cardelli J. A., Clayton G. C., Mathis J. S., 1989, ApJ, 345, 245

Chabrier G., 2003, PASP, 115, 763

Cheung E., et al., 2013, ApJ, 779, 162

Elbaz D., et al., 2007, A\&A, 468, 33

Gavazzi G., et al., 2015, A\&A, 580, A116

González Delgado R. M., et al., 2016, A\&A, 590, A44

Han Y., Han Z., 2014, ApJS, 215, 2

Kauffmann G., et al., 2003, MNRAS, 341, 33

Kennicutt Jr. R. C., et al., 2003, PASP, 115, 928

Kennicutt R. C., et al., 2011, PASP, 123, 1347

Lintott C. J., et al., 2008, MNRAS, 389, 1179

Magdis G. E., et al., 2016, MNRAS, 456, 4533

Maragkoudakis A., Zezas A., Ashby M. L. N., Willner S. P., 2017, MNRAS, 466, 1192

Morrissey P., et al., 2007, ApJS, 173, 682

Noeske K. G., et al., 2007, ApJ, 660, L43 

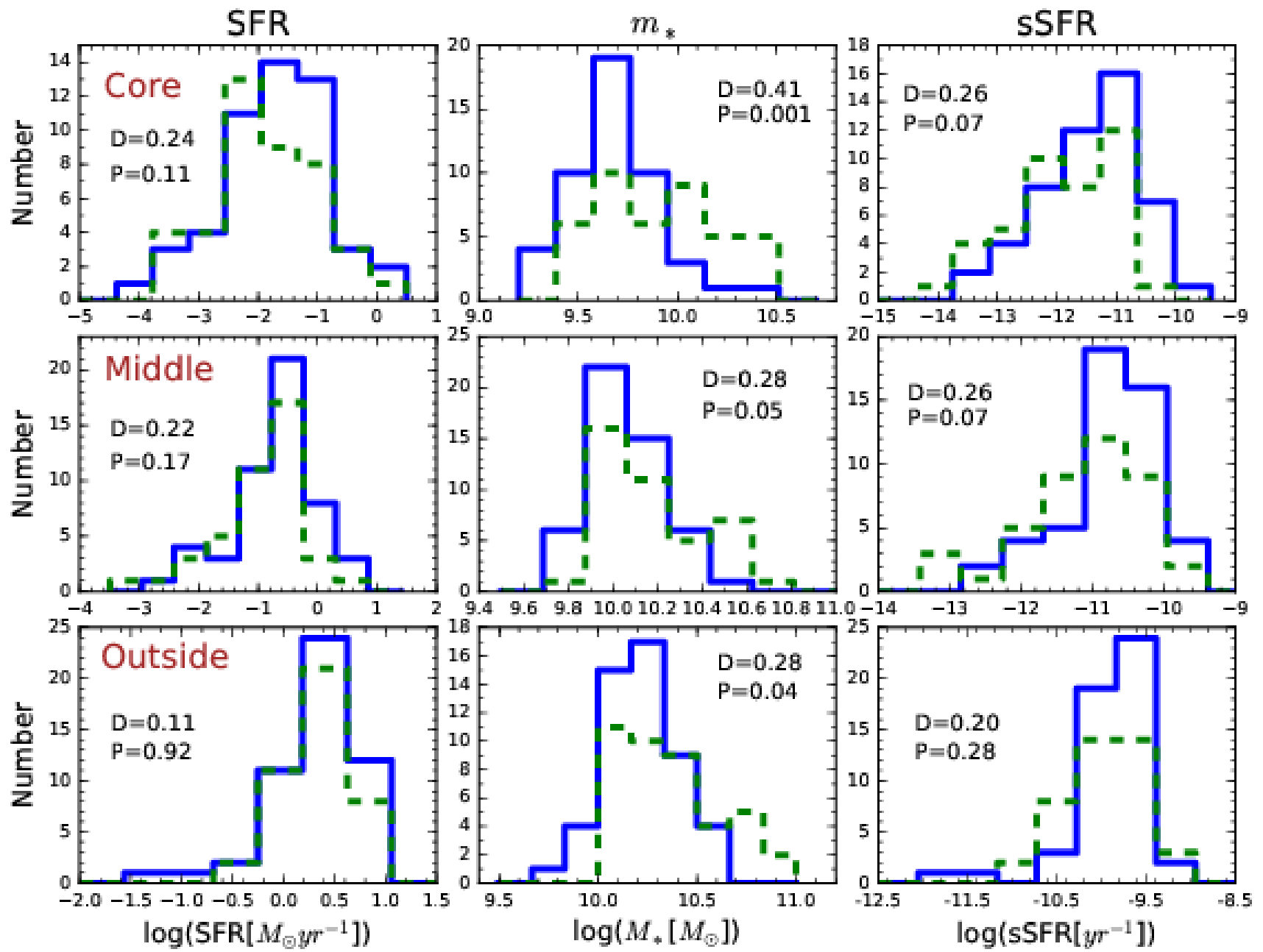

Figure 12. Distributions of SFR, $m_{*}$, and SSFR of barred- and non-barred galaxies in three regions: "core" defined as region inside $10^{-0.5} r_{e}\left(M_{*}\right)$, "middle" defined as $10^{-0.5} r_{e}\left(M_{*}\right)<r \leqslant r_{e}\left(M_{*}\right)$, and "outside" defined as $r>r_{e}\left(M_{*}\right)$. The histograms are shown along with D- and P- values resulted from KS test. Solid blue and dashed green histograms are for non-barred and barred galaxies, respectively.

Pérez E., et al., 2013, ApJ, 764, L1

Renzini A., Peng Y.-j., 2015, ApJ, 801, L29

Rieke G. H., Alonso-Herrero A., Weiner B. J., Pérez-González

P. G., Blaylock M., Donley J. L., Marcillac D., 2009, ApJ, 692,556

Salim S., et al., 2007, ApJS, 173, 267

Sawicki M., 2012, PASP, 124, 1208

Seaton M. J., 1979, MNRAS, 187, 73P

Sorba R., Sawicki M., 2015, MNRAS, 452, 235

Speagle J. S., Steinhardt C. L., Capak P. L., Silverman J. D., 2014, ApJS, 214, 15

Spinoso D., Bonoli S., Dotti M., Mayer L., Madau P., Bellovary J., 2017, MNRAS, 465, 3729

Tremonti C. A., et al., 2004, ApJ, 613, 898

Walcher J., Groves B., Budavári T., Dale D., 2011, Ap\&SS, 331, 1

Welikala N., Connolly A. J., Hopkins A. M., Scranton R., Conti A., 2008, ApJ, 677, 970

Whitaker K. E., van Dokkum P. G., Brammer G., Franx M., 2012, ApJ, 754, L29

Wuyts S., et al., 2012, ApJ, 753, 114

Wuyts S., et al., 2013, ApJ, 779, 135

York D. G., et al., 2000, AJ, 120, 1579
Zhou Z.-M., Cao C., Wu H., 2015, AJ, 149, 1

Zibetti S., Charlot S., Rix H.-W., 2009, MNRAS, 400, 1181

da Cunha E., Charlot S., Elbaz D., 2008, MNRAS, 388, 1595

\section{APPENDIX A: ROBUSTNESS OF OUR METHOD}

In order to evaluate the reliability of the pixel-to-pixel SED fitting method in estimating a local SFR, we perform the pixel-to-pixel SED fitting in the central region of M51, which have resolved MIR dataset from the SINGS survey (Kennicutt et al. 2003). Figure A1 shows the dust reddening tracks of the Calzetti et al. (2000) and Milky-Way dust extinction laws in the colour-colour diagram along with the distribution of M51's photometric data in the bins. Milky-Way dust law have curvy dust extinction tracks in the $F U V-N U V$ vs. $F U V-u$ and $F U V-N U V$ vs. $N U V-u$ colour-colour diagrams which deviate from overall distribution of photometric data, similar to the results shown in Figure 3.

The result of the pixel-to-pixel SED fitting is shown in 


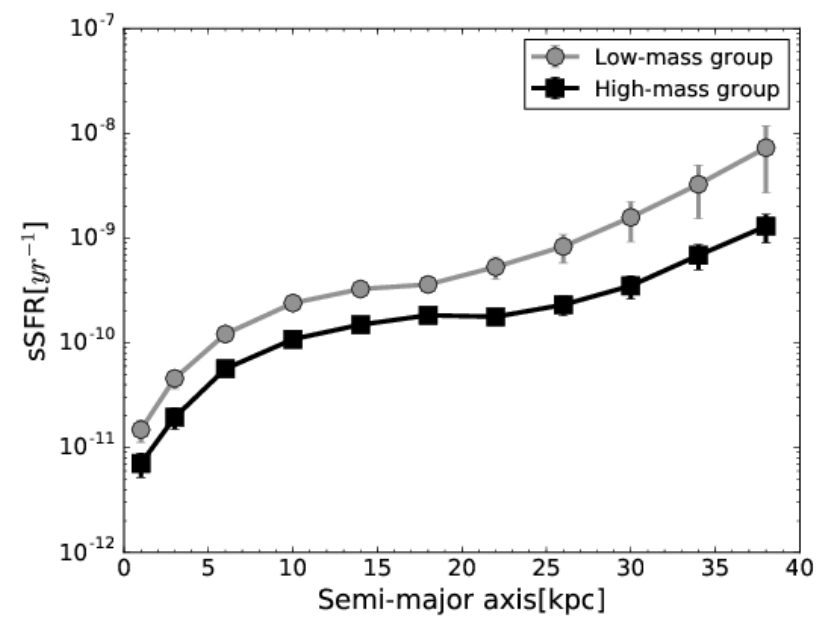

Figure 13. Comparison between the average $\operatorname{sSFR}(r)$ profiles of low- and high-mass groups (grey circle and black square, respectively) in the galaxy sample. The error bars are derived from standard error of mean.

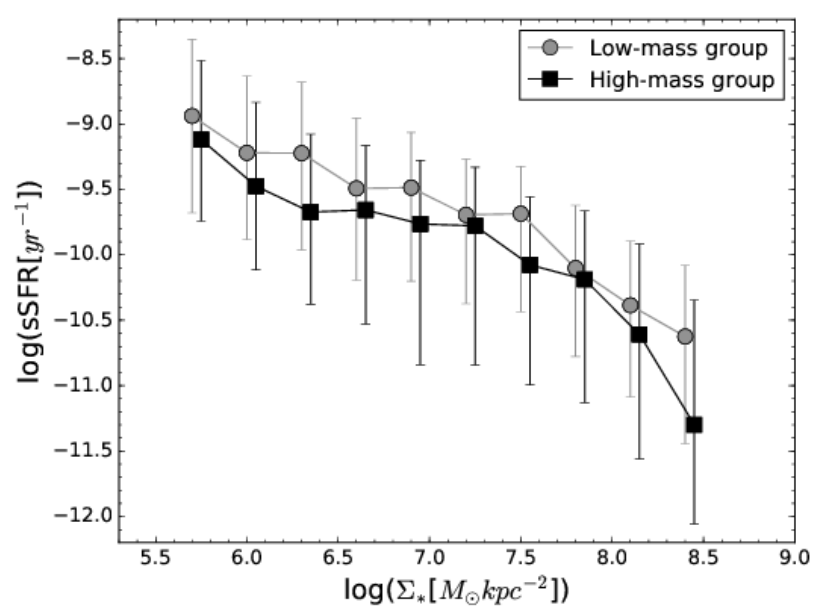

Figure 14. Comparison between the mode sSFR vs. $\Sigma_{*}$ profiles of low- and high-mass groups (grey circle and black square, respectively) in the galaxy sample. For clarity, grey circles are shifted by 0.05 dex to the left.

Figure A2. We check the reliability of our pixel-to-pixel SED fitting method by comparing the SFR estimates by the maximum likelihood fitting with those by the Bayesian method with two different PDFs. Figure A3 shows comparison between M51's spatially-resolved SFR resulted from the SED fitting. The left, middle, and right panels show the results of three different SED fitting method with the maximum likelihood, Bayesian with $P(\theta \mid X)$ with Student's t distribution with $v=3$, and Bayesian with $P(\theta \mid X) \propto \exp \left(-\chi^{2} / 2\right)$. They are compared with SFR estimated from $24 \mu \mathrm{m}$ flux with the SFR-24 $\mu \mathrm{m}$ flux relation in Rieke et al. (2009). We find that the Bayesian method with Student's t distribution with $v=3$ give the best estimate of SFR with smaller scatter from the proportionality. The distribution of the $\log \left(\mathrm{SFR}_{\mathrm{fit}} / \mathrm{SFR}_{24 \mu m}\right)$ has scatter $(\sigma)$ of 0.40 dex and mean value $(\mu)$ of -0.31 dex.

Our new method of the Bayesian SED fitting with Student's t distribution of $v=3$ is also verified by fitting with mock SEDs. We construct mock SEDs generating from Bruzual \& Charlot (2003) with randomly selected parameters $(Z, \tau, E(B-V)$, and age). Some normalizations (randomly selected in the range of $10^{7}$ to $10^{13}$ ) are applied to each $\mathrm{SED}$ then assigned an $\mathrm{S} / \mathrm{N}$ ratio. Adding random noise following gaussian distribution with standard deviation correspond to the flux error from the $\mathrm{S} / \mathrm{N}$ ratio, also applied the Bayesian SED fitting to simulate the observational effect. We fit mock SEDs with Bayesian method with 11 different PDFs: flat (no-weighting), Student's t distribution with $v=0.001,1,3,5,6,8,10,15,20$, and Gaussian. Each fitting is done with two set of models, one with $\tau$ ranging from 0.1 to $10 \mathrm{Gyr}$ and the other with a range of 0.1 to $2 \mathrm{Gyr}$, to test the stability of the method against the choice of model parameter ranges.

Figure A4 shows example of two fitting results, mock SEDs with $\tau$ from 0.1 to 10 Gyr using Bayesian method with Gaussian PDFs (left panel) and Student's t distribution of $v=3$ (right panel). Histogram in the inset shows distributions of $\log (\mathrm{SFR}-$ true/SFR - fit). The distributions indicate that Bayesian method with PDF of Student's t distribution of $v=3$ is better than those with Gaussian PDFs as it has lower systematic offset and scatter. Figure A5 shows means (left panel) and scatters (right panel) of $\log (\mathrm{SFR}$ - true/SFR - fit) from various PDFs. The x-axis represents a various Bayesian fitting method with $v$ indicate the $v$ value of Student's t distribution. Different symbols and colours mean : mock SEDs with random noise fitted to model SEDs with $\tau[0.1: 2]$ (red triangles) and $\tau[0.1: 10]$ (yellow pentagons). The Bayesian method with PDF of Student's t distribution with $v=3$ gives the best result compared to the other PDFs in the fitting tests.

This paper has been typeset from a $\mathrm{T}_{\mathrm{E} X} \mathrm{~L} \mathrm{LT}_{\mathrm{E}} \mathrm{X}$ file prepared by the author. 

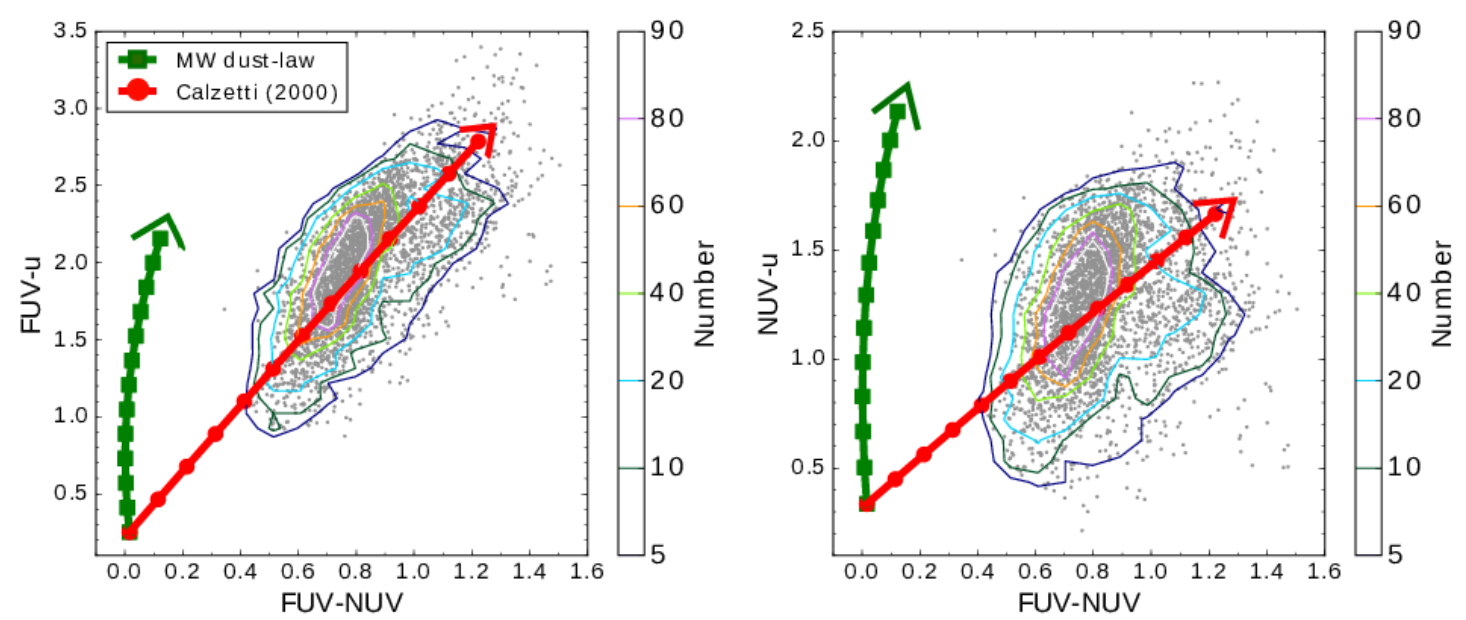

Figure A1. Dust extinction tracks of Calzetti et al. (2000) (red circles) and Milky Way (green squares) dust extinction laws for a model SED with $Z=0.02, \tau=3.7 \mathrm{Gyr}$, and age $=2.5 \mathrm{Gyr}$ in the colour-colour diagrams (left panel: $F U V-N U V$ vs. $N U V-u$, right panel: $F U V-N U V$ vs. $N U V-u$ ) along with the distribution of photometries of the M51's bins. The circles and squares mark increasing colour excess $E(B-V)$ from 0 to 0.6 with a step of 0.05 .
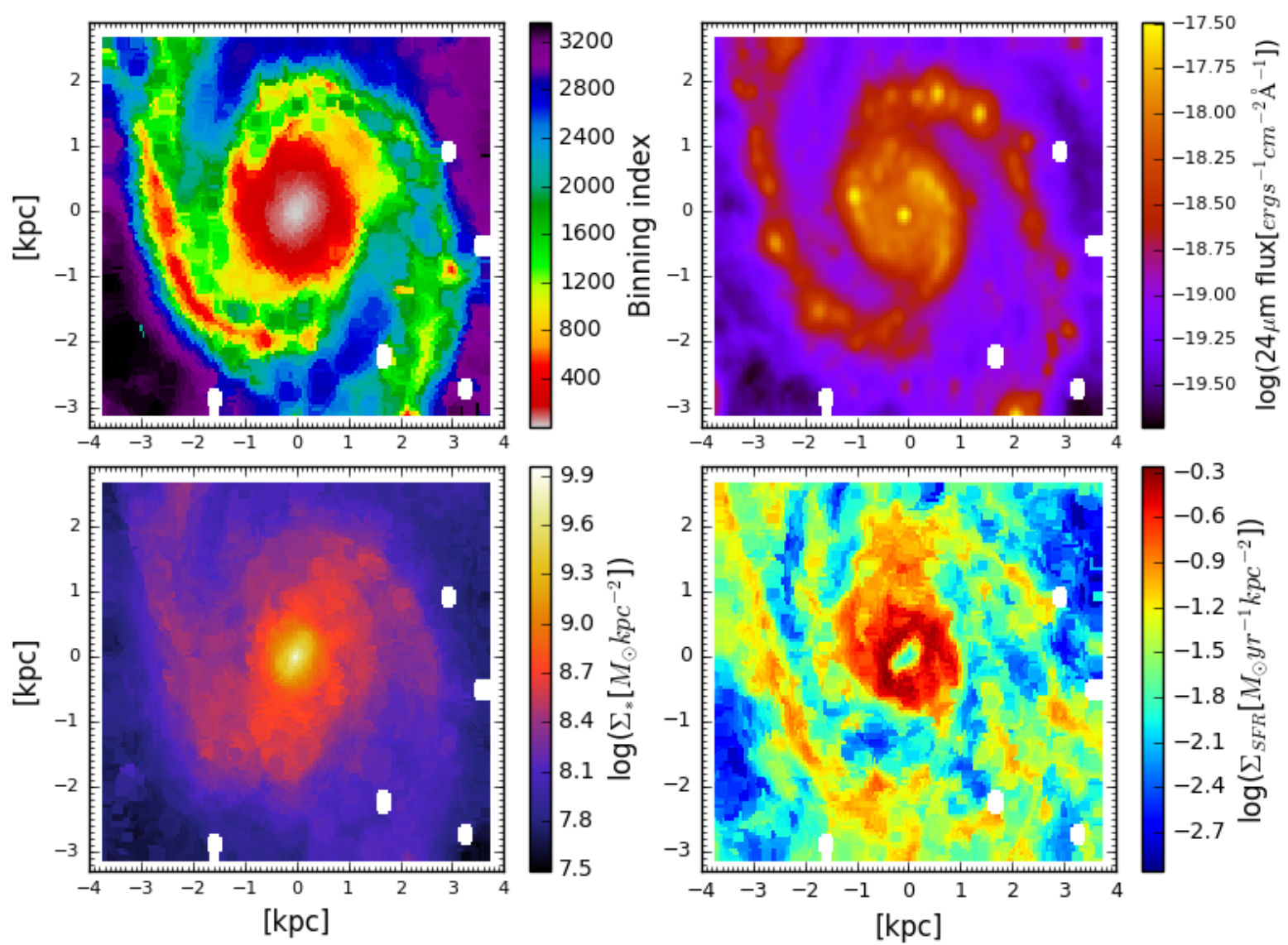

Figure A2. Pixel-to-pixel SED fitting result for M51 galaxy. Clockwise from the top-left panel : pixel binning result, spatially-resolved $24 \mu m$ flux map, spatially-resolved $\Sigma_{\mathrm{SFR}}$ map, and spatially-resolved $\Sigma_{*}$ map. 

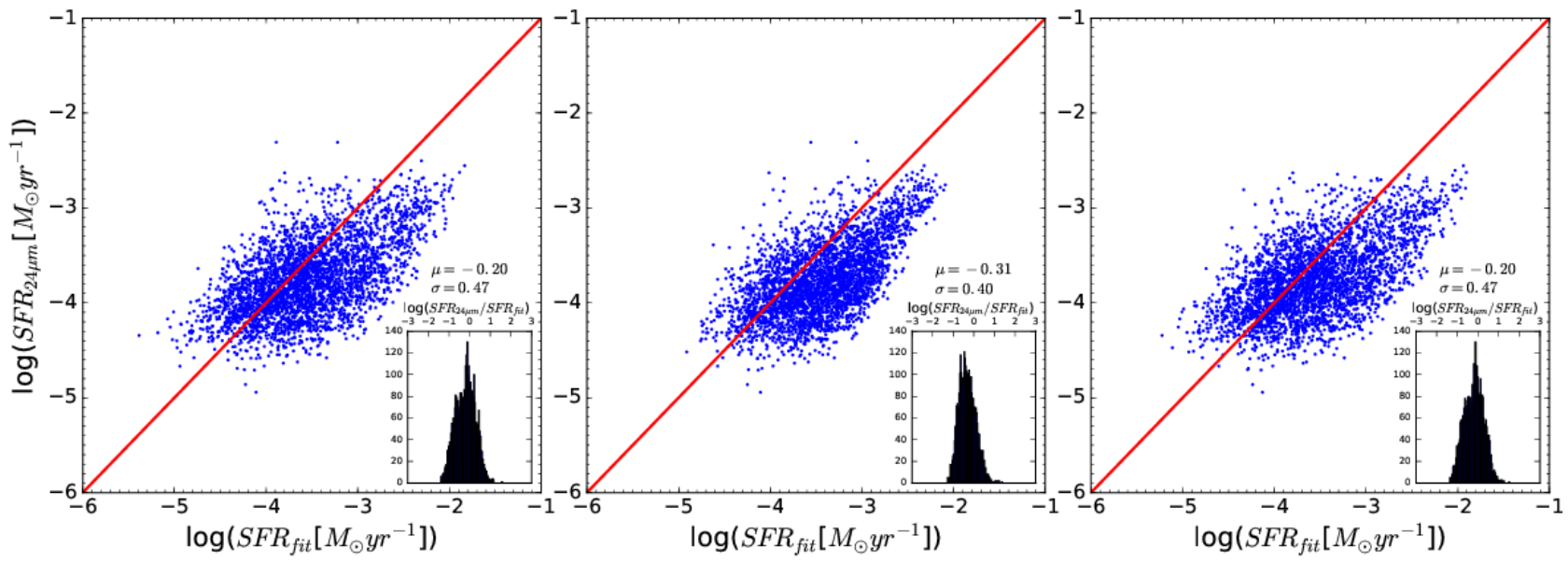

Figure A3. Comparison between three fitting method in term of their consistency with SFR from $24 \mu m$ flux : minimum $\chi^{2}$ (left panel) and Bayesian statistics-based fitting with two different PDFs, $\mathrm{P}(\theta \mid X)$ following Student's t distribution with $v=3$ (middle panel), and $\mathrm{P}(\theta \mid X) \propto \exp \left(-\chi^{2} / 2\right)($ right panel)
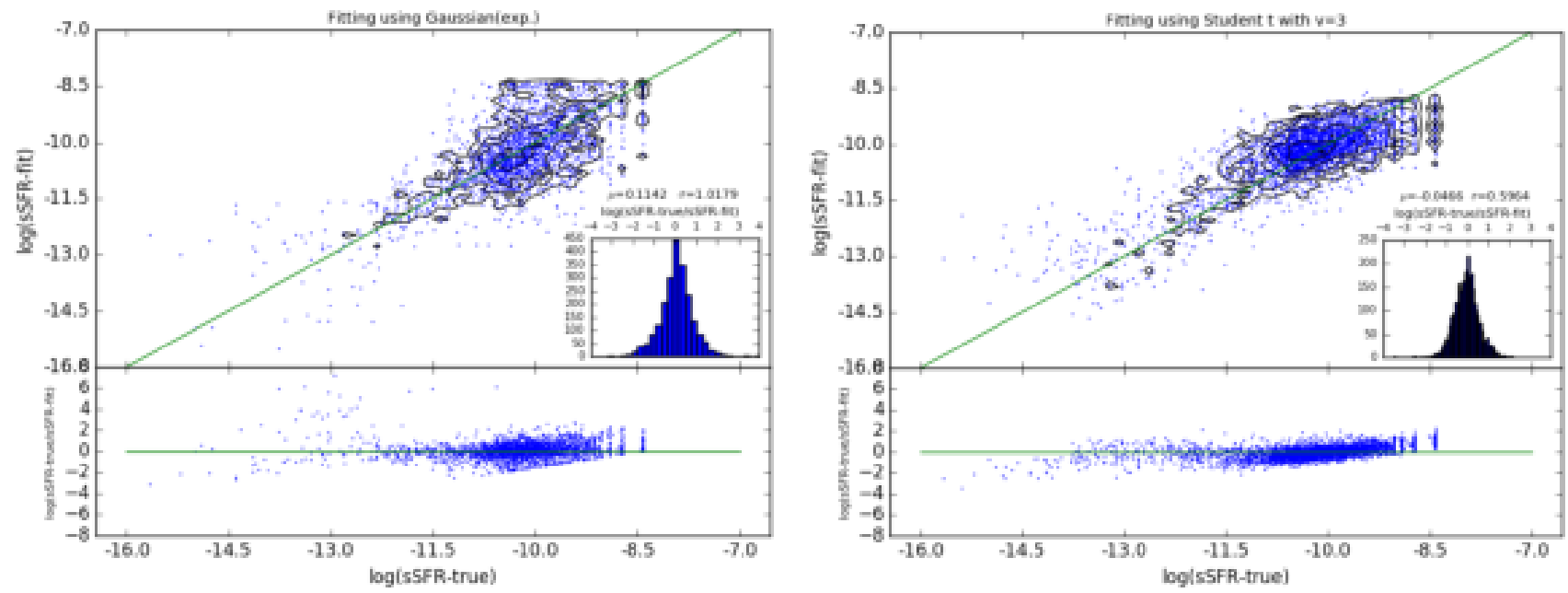

Figure A4. Fitting test with mock SEDs using the Bayesian fitting with $\mathrm{P}(\theta \mid X) \propto \exp \left(-\chi^{2} / 2\right)$ (or gaussian) (left panel) and $\mathrm{P}(\theta \mid X)$ following Student's t distribution with $v=3$ (right panel). 

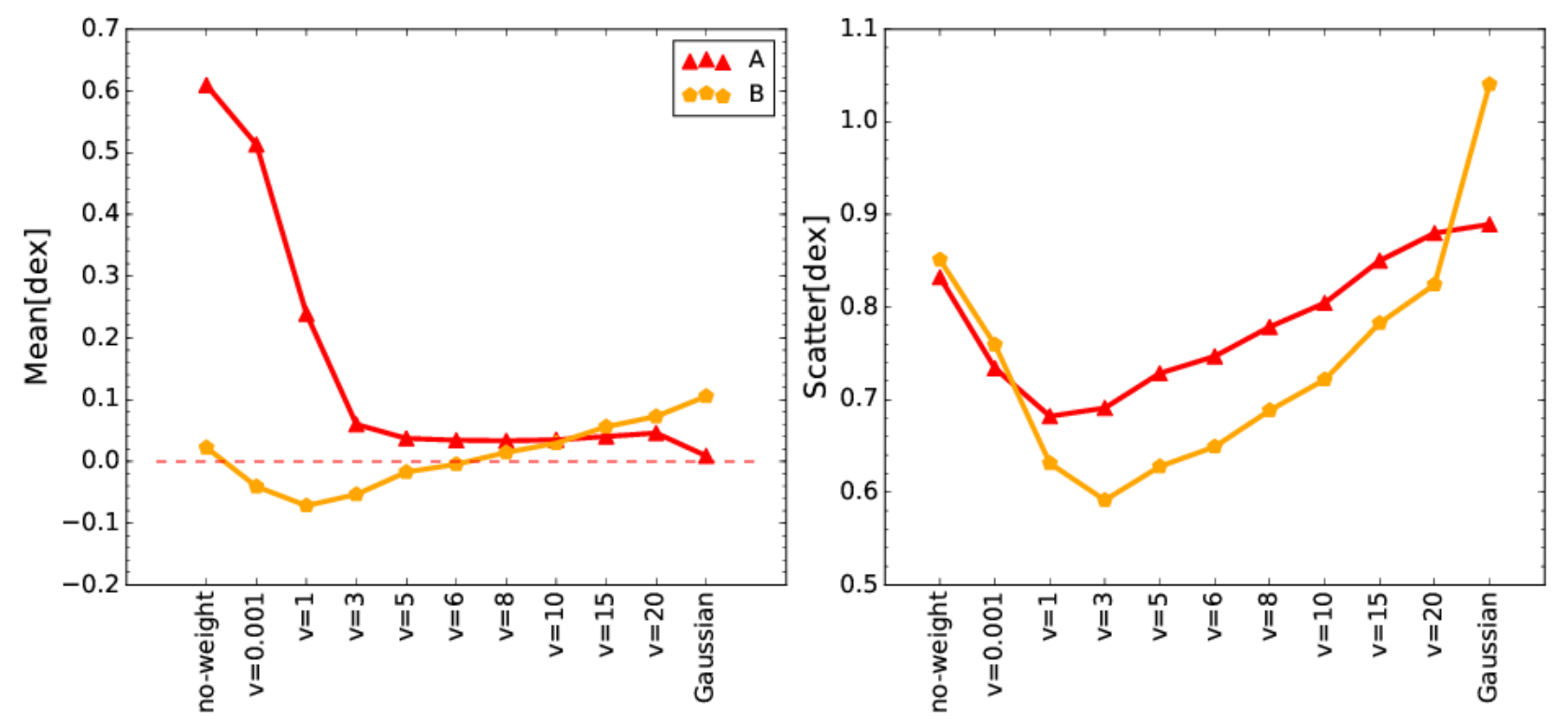

Figure A5. Means (left panel) and scatters (right panel) of $\log (\mathrm{SFR}$ - true/SFR - fit) distributions from several different fitting methods and mock SEDs. Different symbols and colours mean : mock SEDs with random noise fitted to model SEDs with $\tau[0.1: 2]$ (A) and $\tau[0.1: 10]$ (B). $\mathrm{x}$-axis represent Bayesian fitting method. 